\title{
Metabolic and molecular analysis of nonuniform anthocyanin pigmentation in tomato fruit under high light
}

\author{
Yanjie Zhang $\mathbb{B}^{1,2}$, Yan $\mathrm{Li}^{2}$, Wanping $\mathrm{Li}^{2}$, Zongli $\mathrm{Hu}^{1}$, Xiaohui $\mathrm{Yu}^{1}$, Yun $\mathrm{Tu}^{1}$, Min Zhang ${ }^{2}$, Jinyong Huang ${ }^{2}$ and \\ Guoping Chen ${ }^{1}$
}

\begin{abstract}
Pigment intensity and patterns are important factors that determine the nutritional and market values of tomato fruits. The acropetal manner of light-dependent anthocyanin accumulation with the highest levels at the stem end of the fruit makes Pro35S:BrTT8 tomato plants an ideal system for investigating the effects of light intensity on anthocyanin biosynthesis. Extensive transcript analyses indicate that anthocyanin pigmentation in Pro35S:BrTT8 plants under high light might be coordinately regulated by the exogenous protein BrTT8 and endogenous proteins SIAN2 and SIMYBL2. Furthermore, yeast two-hybrid assays showed that BrTT8 could interact efficiently with SIAN2, SIMYBL2, and SIAN11. Moreover, the physical interaction between BrTT8 and SIAN2 was validated by FRET. Simultaneous overexpression of SIAN2 and BrTT8 activated significant anthocyanin biosynthesis in infiltrated tobacco leaves. In addition, the ability of SIMYBL2 to suppress anthocyanin accumulation was also demonstrated in infiltrated tobacco leaves. Altogether, these results prove that tissue-specific assemblage of the heterogeneous MYB-bHLH-WD40 complex consisting of SIAN2, BrTT8 and SIAN11 triggers nonuniform anthocyanin accumulation in tomato fruit under high light. Additionally, it is proposed that a negative-feedback loop fulfilled by SIMYBL2 also participates in the regulation of anthocyanin production.
\end{abstract}

\section{Highlight}

- High-light-induced nonuniform anthocyanin pigmentation in fruits of Pro35S:BrT78 tomato plants is due to regionspecific assemblage of a functional MBW protein complex consisting of BrTT8, SIAN2 and SIAN11.

\section{Introduction}

Anthocyanins are the main water-soluble pigments widespread in many flowering plants. The red, blue, and purple colors found in various plant tissues, including flowers, leaves, fruits, and seeds, are mostly attributed to

\footnotetext{
Correspondence: Yan Li (yanli2018@zzu.edu.cn) or

Guoping Chen (chenguoping@cqu.edu.cn)

'Bioengineering College, Chongqing University, 400030 Chongqing, People's Republic of China

${ }^{2}$ School of Agricultural Sciences, Zhengzhou University, 450001 Zhengzhou,

People's Republic of China
}

the accumulation of anthocyanins ${ }^{1-3}$. In addition to their well-known physiological functions of serving as pollinator and seed disperser attractants, anthocyanins also play essential roles in protecting plant tissues from being damaged by high light, UV radiation, cold, drought, nutrient deficiency, and pathogen attack $^{4}$; anthocyanins also act as important health-promoting supplements in the human daily diet. A growing amount of evidence has shown that regular intake of anthocyanins is associated with a lower risk of artherosclerosis and related diseases due to their ability to inhibit the oxidation of low-density

\section{(c) The Author(s) 2019}

(c) (i) Open Access This article is licensed under a Creative Commons Attribution 4.0 International License, which permits use, sharing, adaptation, distribution and reproduction c. in any medium or format, as long as you give appropriate credit to the original author(s) and the source, provide a link to the Creative Commons license, and indicate if changes were made. The images or other third party material in this article are included in the article's Creative Commons license, unless indicated otherwise in a credit line to the material. If material is not included in the article's Creative Commons license and your intended use is not permitted by statutory regulation or exceeds the permitted use, you will need to obtain permission directly from the copyright holder. To view a copy of this license, visit http://creativecommons.org/licenses/by/4.0/. 
lipids ${ }^{5}$. The health-promoting effects of anthocyanins are considered to be closely linked to high antioxidant activities and the capacity to eliminate reactive oxygen species $^{6-8}$. However, recent studies have shown that anthocyanins and related derivatives are involved in modulating signaling pathways in mammalian cells ${ }^{9}$. In addition, the effects of anthocyanins on the prevention of cancer and some other chronic illnesses have been demonstrated ${ }^{10,11}$.

As the main subclass of phenylpropanoid metabolites, anthocyanins employ a biosynthetic pathway that has been well characterized (Fig. 1). The synthesis of cinnamic acid catalyzed by phenylalanine ammonia-lyase (PAL) represents the initial reaction step of the phenylpropanoid pathway ${ }^{12}$. Chalcone synthase (CHS) catalyzes the condensation reaction with 4-coumaroyl $\mathrm{CoA}$ and malonyl $\mathrm{CoA}$ as substrates to produce naringenin chalcone, the entry molecule of the flavonoid pathway. 4-Coumaroyl CoA can be esterified with quinic acid by hydroxycinnamoyl CoA-quinate transferase (HQT), leading to the production of chlorogenic acid (CGA), a typical phenolic acid widespread in Solanaceae plants. The basic skeleton of all flavonoids (including anthocyanins, chalcones, flavones, flavonols, flavandiols, and condensed tannins) consists of three aromatic rings generated by $\mathrm{CHS}$ and $\mathrm{CHI}$. F3H catalyzes the oxidation of the central ring of naringenin, yielding dihydrokaempferol. Moreover, dihydrokaempferol can be further hydroxylated on the $3^{\prime}$ or $5^{\prime}$ position of the B-ring by flavonoid $3^{\prime}$-hydroxylase $\left(\mathrm{F} 3^{\prime} \mathrm{H}\right)$ and/or flavonoid $3^{\prime} 5^{\prime}$-hydroxylase $\left(\mathrm{F}^{\prime} 5^{\prime} \mathrm{H}\right)$, resulting in the production of dihydroquercetin and dihydromyricetin, respectively ${ }^{2}$. Additionally, cyanidin and delphinidin can be further methylated at the $3^{\prime}$ or $5^{\prime}$ position of the B-ring by flavonoid 3', 5'-methyltransferase (FAOMT), resulting in the production of peonidin, petunidin and malvidin, respectively. Unstable anthocyanidins are modified mostly by glycosylation and converted into stable forms, namely, anthocyanins ${ }^{2}$. Then, the final productions are transported into vacuoles coordinately by multidrug and toxic compound extrusion (MATE) transporters, glutathione S-transferase (GST) or ABC transporters ${ }^{13,14}$. Except for anthocyanins, chalcones and CGA, flavonols and flavones can also be produced in the branch pathway of phenylpropanoid metabolism.

The biosynthesis of phenylalanine-derived compounds, especially flavonoids, is mainly regulated by MYB proteins, which constitute the largest class of modulators for secondary metabolism ${ }^{15,16}$. A total of 339 and 230 MYB proteins were characterized in Arabidopsis thaliana and rice, respectively ${ }^{17}$. Since structurally similar R2R3-MYB TFs were able to trigger identical target genes by binding with the same cis elements in promoters, functional redundancy for these MYB proteins has often been indicated $^{18,19}$. By analyzing mutant plants with abnormal levels of anthocyanin production, many modulators were cloned and characterized, including basic helix-loop-helix (bHLH) TFs, WD40 repeat (WDR) proteins, basic Leucine Zipper (bZIP) TFs, WRKY TFs, MADS-box TFs and SQUAMOSA PROMOTER BINDING PROTEIN (SPL) $\mathrm{TFs}^{20-23}$. Further studies prove that structural genes catalyzing the late step reactions of the anthocyanin pathway are regulated directly by a ternary protein complex consisting of R2R3-MYB TFs (like PAP1, PAP2 MYB113, and MYB114 in Arabidopsis), bHLH TFs (like TT8, GL3, and EGL3), and WD40 repeat proteins (such as TTG1) $)^{21,24,25}$.

Among all the regulators mentioned above, MYB and bHLH proteins are thought to be the key factors that regulate spatial and temporal anthocyanin accumulation in plants ${ }^{9,17,26}$. It has been demonstrated that AtMYB4, an R2R3-MYB repressor, could repress the transcription of target gene $\mathrm{C} 4 \mathrm{H}$ by a special motif in the C-terminal domain ${ }^{27}$. In subsequent studies, several anthocyanin biosynthesis repressors from the R2R3 and R3-MYB subfamilies have been identified and characterized. FaMYB1 and PhMYB27, belonging to the R2R3-MYB subfamily, have been identified as transcriptional repressors in strawberry ${ }^{28}$. In addition, small R3-MYB transcription factors that inhibit anthocyanin biosynthesis by acting as competitive inhibitors of the R2R3-MYB activators have also been cloned $^{29-31}$. In Arabidopsis, CAPRICE (CPC), an R3-MYB protein was identified as a determinant of cell differentiation at first and was proved to be a transcriptional repressor in anthocyanin biosynthesis in transgenic tobacco and tomato plants in further studies ${ }^{29,31}$. In addition, SlTRY, an ortholog gene of TRYPTICHON (TRY), was identified, and the ectopic expression of SITRY significantly reduced anthocyanin accumulation in transgenic Arabidopsis ${ }^{31}$. In the latest study, a 4-bp insertion in the coding region of the atv (atroviolacium) locus encoding a putative R3-MYB repressor significantly enhanced anthocyanin accumulation in tomato fruits ${ }^{32,33}$.

Except for endogenous signals, exogenous stimulation factors, such as high light, drought, cold and phosphorus deficiency, operate coordinately to accurately regulate the anthocyanin pathway ${ }^{34-37}$. In previous studies, several bHLH proteins have been introduced into Arabidopsis, tomato, Nicotiana and petunia for functional analysis in anthocyanin biosynthesis regulation ${ }^{30,38-41}$. In addition, light-induced anthocyanin accumulation was also carefully examined in the vegetative tissues of Pro35S: $\mathrm{ZmLc}$ petunia plants ${ }^{35}$. Moreover, SlTT8 (also named SlAN1 and $S l A H)$ was shown to participate in the regulation of anthocyanin biosynthesis and is induced by low temperature $^{32,41,42}$. However, high-light-induced anthocyanin pigmentation in tomato fruits has not been reported or studied. Pro35S:BrTT8 plants created in our previous 


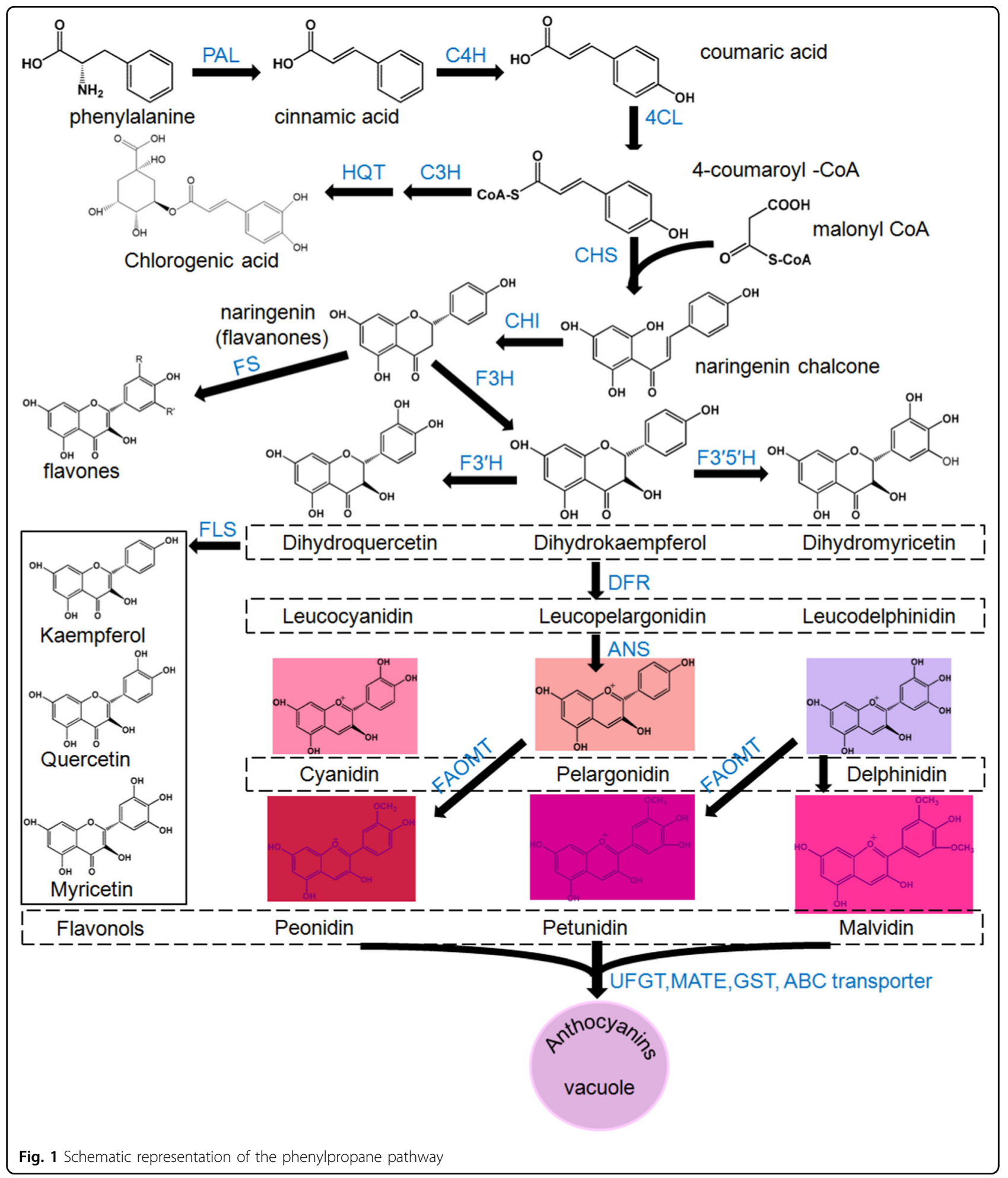

work displayed abundant anthocyanin accumulation in both vegetative and fruit tissues when grown in natural high-light conditions (open field) but remained anthocyaninless under low-light conditions (greenhouse) ${ }^{44}$.
Interestingly, anthocyanin accumulates in a unique pattern in Pro35S:BrTT8 fruits under natural high-light conditions. As tomato (Solanum lycopersicum L.) is an ideal model system for studying fleshy fruit 
development ${ }^{43}$. Pro35S:BrTT8 tomato plants serve as an excellent model system for the study of nonuniform anthocyanin pigmentation in fruits exposed to high light.

\section{Materials and methods Chemicals and reagents}

Ultrapure water was prepared by a Milli-Q purification system. HPLC grade methanol, acetonitrile, acetic acid, formic acid, neochlorogenic acid, chlorogenic acid, cryptochlorogenic acid, kaempferol, and delphinidin-3-Orutinoside were purchased from Sigma-Aldrich.

\section{Plant material and growth conditions}

Diploid tomato plants (Solanum lycopersicum Mill. cv Ailsa Craig, AC) were generously provided by Dr. Donald Grierson of the School of Biosciences, University of Nottingham, $\mathrm{UK}^{44}$. The Pro35S:BrTT8 transgenic tomato seeds were generated from the primary transformants of $\mathrm{AC}$ in our previous article ${ }^{45}$. In this study, the existing photoperiod was $16 \mathrm{~h}$, and the temperature in the greenhouse was maintained between 20 and $28^{\circ} \mathrm{C}$. In addition, the relative humidity was approximately $60 \%$. Plants were cultured under low-light conditions to inhibit anthocyanin accumulation until they exhibited approximately six nodes. All of the axillary buds were cut to prevent a branched architecture, which may result in self-shading effects. Six replicates of both AC and Pro35S:BrTT8 plants were transferred to either artificial low-light or high-light conditions. The seedlings were maintained for 10 days under each light treatment. After that, leaves were sampled and frozen immediately in liquid nitrogen and kept at $-80^{\circ} \mathrm{C}$ for pigment extraction and RNA isolation.

In the following experiments investigating nonuniform anthocyanin pigmentation in Pro35S:BrTT8 fruits, 18 homozygous (six individuals for each independent transgenic line) plants and wild-type plants were grown in a greenhouse under artificial high-light conditions. Fruits were covered by translucent plastic bags after pollination to simulate low-light conditions. Epicarps and mesocarps were removed and sampled by hand, frozen immediately in liquid nitrogen and kept at $-80^{\circ} \mathrm{C}$ for pigment extraction and RNA isolation.

\section{Light treatments}

The high-light treatment was provided by supplementing normal light with a metal-halide lamp (Philips) under a short-day ( $8 \mathrm{~h}$ of illumination) photoperiod, and plants were arranged randomly to prevent shading. The light level varied between 50 and $100 \mu \mathrm{mol} \mathrm{m}^{-2} \mathrm{~s}^{-1}$ in the lowlight conditions, while the light intensity was constant at $700 \mu \mathrm{mol} \mathrm{m}^{-2} \mathrm{~s}^{-1}$ in the artificial high-light conditions. Unless otherwise stated, samples were all collected at the end of the light period of each day. Light measurements were determined using a digital lux meter sensor.

\section{Secondary metabolite extraction and HPLC-ESI-MS/MS analysis}

Leaf tissues for anthocyanin measurements were collected from nodes 4-6 from each plant at the end of the light period of each day to standardize any circadian or diurnal effects on the transcript abundance of anthocyanin biosynthetic genes. Pigments were extracted and analyzed with a previously reported method of HPLCESI-MS/MS ${ }^{46}$. Anthocyanin levels were determined as delphinidin-3-O-rutinoside equivalents by integrating peak areas. Similarly, kaempferol-3-O-rutinoside-7-Oglucoside levels were determined as kaempferol equivalents. HPLC separation was achieved using ACQUITY UPLC $^{\text {max }}$ Xbridge BEH C18 columns $(150 \times 2.1 \mathrm{~mm}, 3.5$ $\mu \mathrm{m})$. Both the positive-ion $\left(\mathrm{ESI}^{+}\right)$and negative-ion $\left(\mathrm{ESI}^{-}\right)$ modes were used in the MS system (Xevo G2-XS QTof, Waters Corp., Milford, MA, USA).

\section{Total anthocyanin analysis}

The spectrophotometric differential $\mathrm{pH}$ method was applied for the measurement of total anthocyanins. Frozen samples $(100 \mathrm{mg})$ were crushed into powder in liquid nitrogen and then extracted for further analysis ${ }^{46}$. The total anthocyanin contents of different samples were analyzed in triplicate.

\section{Measurement of chlorophyll}

For the measurement of chlorophyll, a section of fruit pericarp was cut, weighed and ground into powder in a mortar with liquid nitrogen, and then total chlorophyll was extracted and examined with the method reported previously ${ }^{47}$.

\section{RNA extraction and quantitative real-time PCR analysis}

Total RNA extraction and RT-qPCR were carried out as previously reported. Gene expression analysis in tomato plants was normalized to $S l C A C$ as a reference gene $^{48}$. Gene expression analysis in tobacco leaves was normalized to NbActin as a reference gene ${ }^{49}$. The values reported here represent three biological repeats for each sample.

\section{Plasmid construction and tomato transformation}

The full-length open reading frame of SlAN2 cDNA from tomato was amplified using primers SIAN2F (5'GCTCTAGAATGAATACTCCTATGTGTG-3') and SlAN2R (5'-CGCAGAGCTCTTAATTAAGTAGATTCC ATAA- $\left.3^{\prime}\right)$. The amplification products were digested and inserted into the pBI121 plasmid to yield the overexpression plasmid Pro35S:SlAN2. The construction of the overexpression plasmid Pro35S:BrTT8 was described in a previous article ${ }^{45}$. In addition, the overexpression vector Pro35S:SIMYBL2 was generated by the same method with primers SIMYBL2F (5'-GCTCTAGAATG 
AGAAAGCCTTGTTGTG-3') and SIMYBL2R (5'-CGC AGAGCTCCGAGAATGTCTTCGATACT-3'). Subsequently, all the final constructs were sequenced and introduced into wild-type tomatoes using the procedure described previously ${ }^{45}$. Stable transformed plants were selected on the basis of kanamycin resistance and genotyping.

\section{Yeast two-hybrid assay}

To avoid self-activation, the carboxyl terminal-deleted forms of BrTT8 and SITT8 (also named SIAN1 or AH) were excised by EcoRI/SalI double digestion and subcloned into pGBKT7 to generate an in-frame fusion with the GAL4 DNA binding domain, respectively $^{32,33,41,42}$. Full-length sequences of SlAN2, SlMYBL2, SlAN11 and BrTT8 were excised using EcoRI/ $X$ XoI double digestion and subcloned into pGADT7 to generate an in-frame fusion with the GAL4 activation domain. The yeast two-hybrid assays were performed using the Matchmaker ${ }^{\mathrm{TM}}$ Gold Yeast Two-Hybrid System according to the manufacturer's instructions (Clontech, Palo Alto, CA, USA).

\section{FRET measurements}

The FRET efficiency between two fusion proteins, BrTT8-EGFP and SIAN2-mCherry, was measured in the epidermal cells of Nicotiana benthamiana cultivated in the greenhouse as described, and transient Agrobacterium tumefaciens-mediated gene expression was performed as described $^{50}$.

\section{Transient assay}

The open reading frames of BrTT8, SIAN2, SIMYBL2, EGFP, and mCherry were amplified with specific primers (Table S3) and cloned into pBI121 to obtain the expression vectors Pro35S:BrTT8-eGFP, Pro35S:SlAN2-mCherry, Pro35S:SIMYBL2-mCherry and Pro35S:SIMYBL2, respectively. Transient assays were conducted as previously reported $^{50}$. Final measurements of secondary metabolites were performed four days after infiltration.

\section{Sequence analyses}

A multiple sequence alignment was generated with DNAMAN version 5.2.2. A neighbor-joining tree was produced with 1000 bootstrap replicates using the program MEGA (Molecular Evolutionary Genetics Analysis) version 3.1.

\section{Statistical calculations}

Unless otherwise indicated, all data were analyzed by one-way ANOVA, followed by Duncan's multiple range test using the SPSS 17 program (SPSS 17.0, SPSS Inc., USA). Different letters indicate that the results were statistically significant at $P<0.05$.

\section{Results}

Extensive anthocyanin accumulation in Pro35S:BrTT8 tomato plants under natural high-light conditions

In wild-type tomato plants cultivated in natural highlight conditions (700-2000 $\mu \mathrm{mol}$ photons $\mathrm{m}^{-2} \mathrm{~s}^{-1}$ ), only a tiny amount of anthocyanins was observed in the epidermal cells of leaf veins. In contrast, anthocyanin pigmentation occurred widely in the leaves, stems, petals and fruits of Pro35S:BrTT8 tomato plants (T0 generation) (Fig. 2a-h). Two types of glycosylated anthocyanins were separated and identified from the epicarps of Pro35S: BrTT8 fruits (Supplemental Figure S1). Delphinidin-3-Oglucoside-5-O-rutinoside, which occupied $88 \%$ of the total amount of anthocyanins, is indicated by the red frame in Fig. 2 (Table 1). The structures and major cleavage sites of delphinidin-3-O-glucoside-5-O-rutinoside and delphinidin-3-O-glucoside-5-O-( $p$-coumaroyl)arabinoside, confirmed by mass spectrometry, are indicated with dashed arrows (Supplemental Figure S1). Apparently, delphinidin-based anthocyanins occupied the absolute number of percentages, indicating a specific biosynthetic pathway in tomato. Gene expression analysis indicates that BrTT8 promotes anthocyanin production probably by transcriptional activation of structural genes in leaves exposed to natural high light (Supplemental Figure S2).

Compared with natural high-light conditions, Pro35S: BrTT8 tomato plants exhibited an anthocyaninless phenotype under low-light conditions $(50-100 \mu \mathrm{mol}$ photons $\mathrm{m}^{-2} \mathrm{~s}^{-1}$ ) (Fig. 2i). To investigate the effects of light intensity on anthocyanin pigmentation in tomato plants, seedlings of wild-type and Pro35S:BrTT8 (T1 generation) plants were grown under low-light conditions and then treated with artificial high light (700 $\mu$ mol photons $\mathrm{m}^{-2} \mathrm{~s}^{-1}$ ). Compared with anthocyaninless seedlings of wild-type and transgenic tomato plants under low-light conditions, anthocyanin production was enhanced significantly in seedlings under high-light conditions, especially in Pro35S:BrTT8 tomato plants (Fig. 2i, j). In particular, the leaves of Pro35S:BrTT8 tomato plants exposed to high light contained a high concentration of anthocyanins $\left(0.56 \mathrm{mg} \mathrm{g}^{-1} \mathrm{FW}\right)$, while wild-type seedlings under the same conditions displayed a much lower intensity of anthocyanin pigmentation $(<0.13$ $\mathrm{mg} \mathrm{g}^{-1}$ FW) (Fig. 2j).

\section{Transcript changes of anthocyanin biosynthetic and regulatory genes in Pro35S:BrTT8 seedlings under different light conditions}

The expression levels of almost all anthocyanin biosynthetic genes were clearly increased in both transgenic and wild-type plants (HT and $\mathrm{HC}$ ) under artificial highlight conditions compared with plants under low-light conditions (LT and LC) (Fig. 3a). As reported before, the increase of gene transcripts is often congruously 

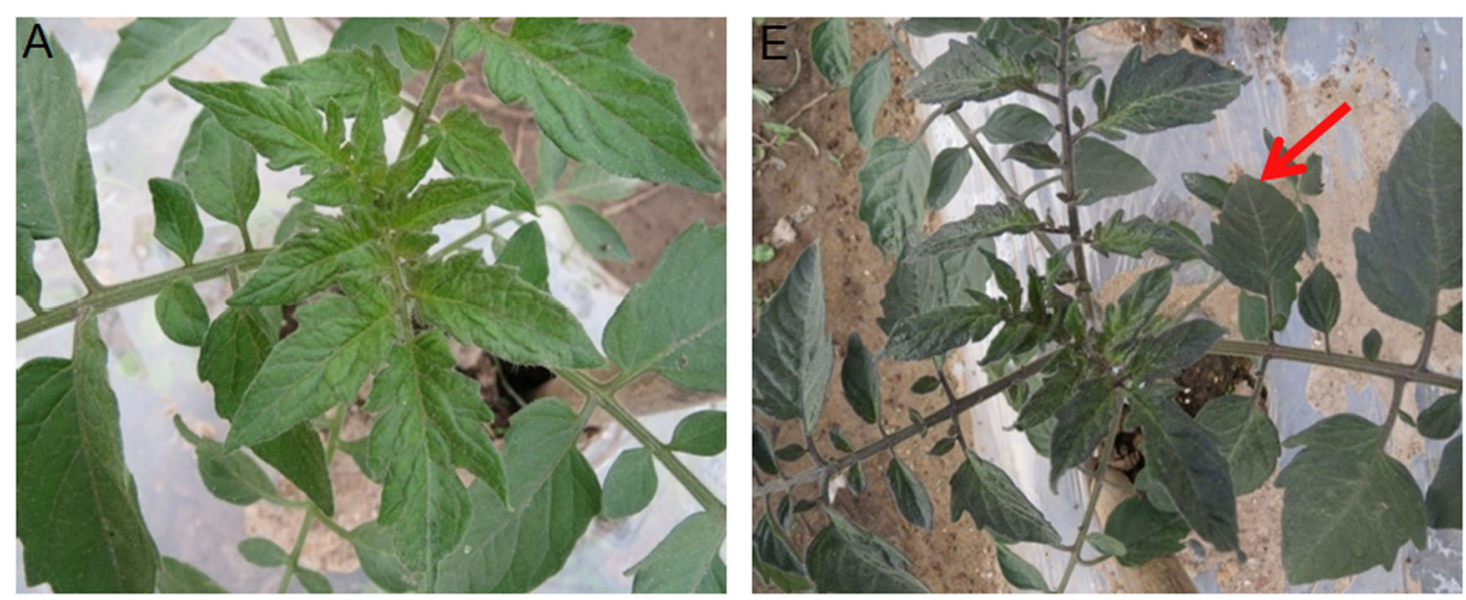
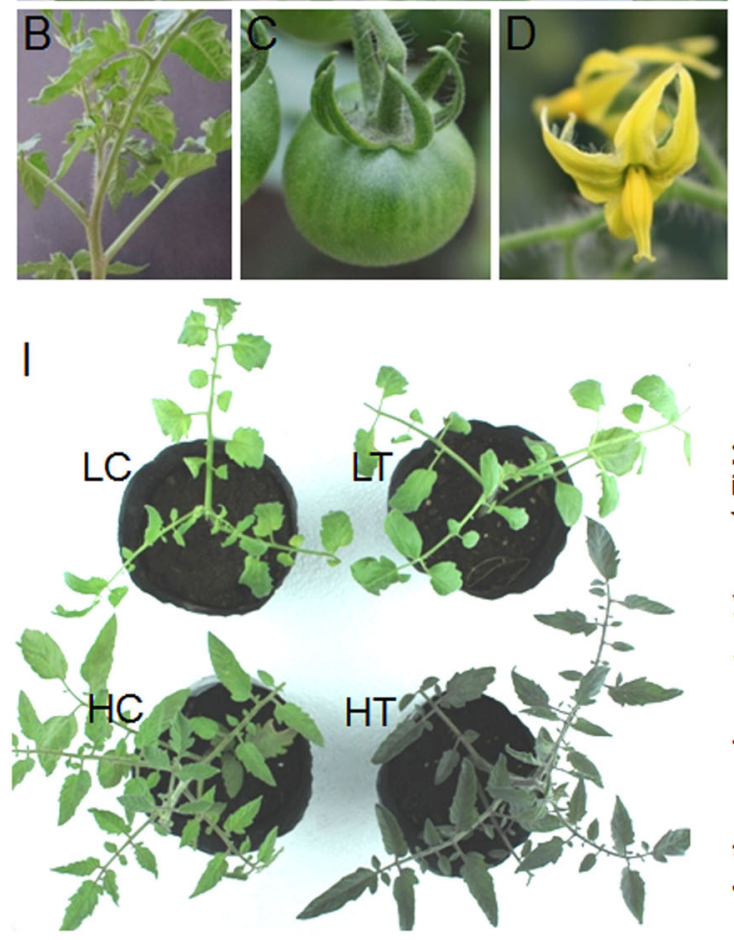
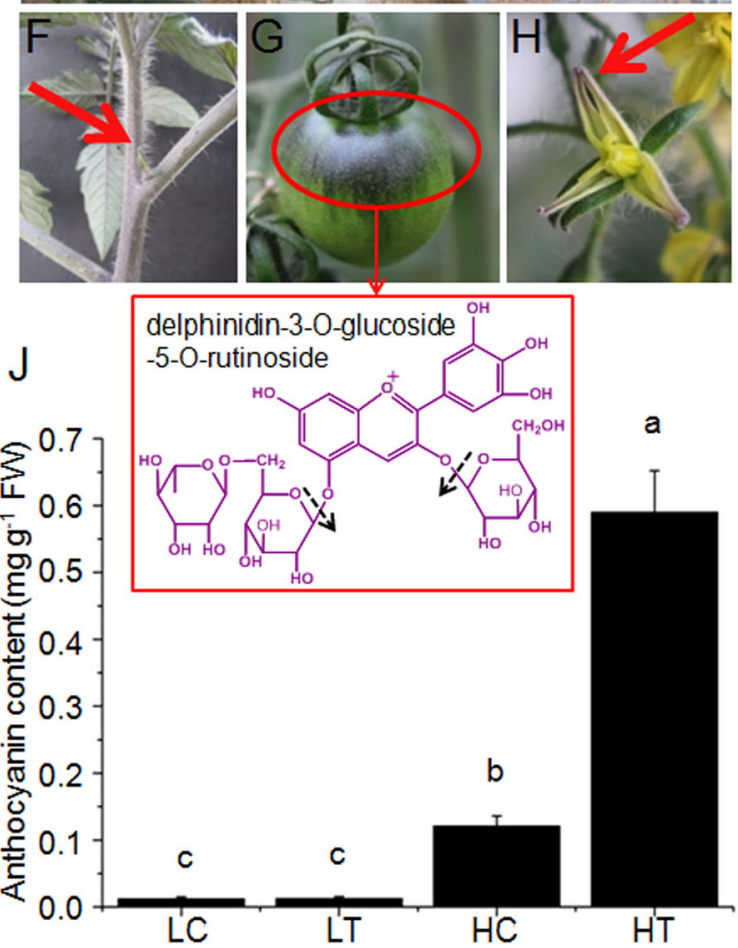

Fig. 2 Anthocyanins accumulated widely in various tissues of Pro35S:BrTT8 tomato plants grown under high-light conditions.

Anthocyanins accumulated considerably in the foliage (e), stems (f), fruits $(\mathbf{g})$ and flowers (h) of Pro35S:BrTT8 plants, with wild-type (AC) plants grown under natural high-light conditions $\left(700-2000 \mu \mathrm{mol}\right.$ photons $\left.\mathrm{m}^{-2} \mathrm{~s}^{-1}\right)$ as a control $(\mathbf{a}-\mathbf{d})$. The structure of the major anthocyanin, delphinidin-3-Oglucoside-5-O-rutinoside, identified in fruits is framed by a red box, and the major cleavage sites confirmed by mass spectrometry are indicated with

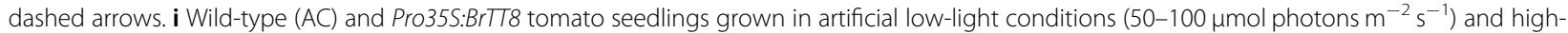
light conditions $\left(700 \mathrm{\mu mol}\right.$ photons $\left.\mathrm{m}^{-2} \mathrm{~s}^{-1}\right)$ exhibit significant differences in anthocyanin accumulation. $\mathbf{j}$ Analysis of total anthocyanin contents in leaves of wild-type and Pro35S:BrT78 tomato seedlings under artificial low-light and high-light conditions. The individual plants shown are representative of the six plants grown per treatment. LC seedlings of control (AC) under low-light conditions, HC seedlings of control under highlight conditions, LT seedlings of transgenic (Pro35S:BrTT8) plants under low-light conditions, HT seedlings of transgenic plants under high-light conditions. Biological replicates were performed in triplicate, and different letters indicate a significant difference at $P<0.05$

paralleled by respective protein changes ${ }^{16}$, which then lead to large amounts of metabolite production. Anthocyanin biosynthetic genes, including $P A L, C 4 H, 4 C L$, CHS1, CHS2, CHI, F3H, F3'H, F3' $5^{\prime} H, D F R, A N S$ and $U F G T$, showed high transcript abundance in wild-type and Pro35S:BrTT8 seedlings under high-light conditions and very weak signals for $F 3^{\prime} H, F 3^{\prime} 5^{\prime} H, D F R$ and $U F G T$ under low-light conditions. These results indicate that the expression of most structural genes was dramatically induced by high light. Specifically, $\mathrm{F}^{\prime} 5^{\prime} \mathrm{H}$ and DFR exhibited the highest fold increases in transgenic plants under high-light exposure (Fig. 3a). On the whole, 
Table 1 Anthocyanin contents ( $\mathrm{mg} \mathrm{g}^{-1}$ dry wt.) in the epicarps of wild-type and Pro35S:BrTT8 fruits under natural highlight conditions $(n=3)$

\begin{tabular}{|c|c|c|c|c|c|c|c|}
\hline \multirow[t]{2}{*}{ No. $^{a}$} & \multirow[t]{2}{*}[\mathrm{M}]{$^{+}(\mathrm{m} / \mathrm{z})$} & \multirow[t]{2}{*}{ MS/MS (m/z) } & \multirow[t]{2}{*}{ Anthocyanins } & \multicolumn{2}{|c|}{ Low light } & \multicolumn{2}{|c|}{ High light } \\
\hline & & & & $W T^{b}$ & Pro35S:BrTT8 ${ }^{\mathrm{c}}$ & WT & Pro35S:BrTT8 \\
\hline 1 & 773 & $465 / 303$ & Delphinidin-3-O-glucoside-5-O-rutinoside & $\mathrm{Nd}^{\mathrm{d}}$ & $\mathrm{Nd}$ & $\mathrm{Nd}$ & $0.53 \pm 0.08$ \\
\hline 2 & 743 & $465 / 303$ & Delphinidin-3-O-glucoside-5-O-(p-coumaroyl)arabinoside & $\mathrm{Nd}$ & $\mathrm{Nd}$ & $\mathrm{Nd}$ & $0.07 \pm 0.02$ \\
\hline Total & & & & $\mathrm{Nd}$ & $\mathrm{Nd}$ & $\mathrm{Nd}$ & $0.60 \pm 0.09$ \\
\hline
\end{tabular}

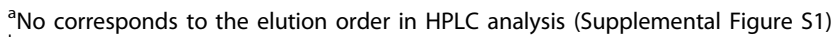

bWT indicates wild type

'Pro35S:BrTT8 indicates transgenic tomato plants with a high expression level of the heterologous gene BrTT8 driven by the $35 \mathrm{~S}$ promoter

${ }^{\mathrm{d}} \mathrm{Nd}$ indicates that no anthocyanin was detected

transgenic plants showed significantly enhanced expression for most of the biosynthetic genes, including CHS1, CHS2, CHI, F3H, F3'H, F3'5'H, DFR, ANS and UFGT, compared with wild-type plants under high-light conditions. However, the expression levels of $P A L, C 4 H$ and $4 C L$, which encode enzymes catalyzing the first three steps of the anthocyanin biosynthetic pathway, did not show obvious differences between wild-type and transgenic plants under high-light conditions. In addition, BrTT8 transcripts were shown to be constantly expressed under both high-light and low-light conditions (Supplemental Figure S3).

All the regulatory genes examined were expressed at similar background levels in Pro35S:BrTT8 and wild-type plants under low-light conditions, and the transcripts of most transcription factors remained relatively constant among samples of HT, HC, LT and LC (Fig. 3b). Nonetheless, the expression of SlAN2 and SlTT8 was enhanced significantly by high light (Fig. 3b). Furthermore, the gene expression of SlTT8 in Pro35S:BrTT8 plants was evidently higher than that of wild-type plants, indicating that BrTT8 further enhanced the expression of SITT8 under high-light treatment. Unexpectedly, the expression of SIMYBL2, a putative transcription repressor, was also upregulated significantly in Pro35S:BrTT8 plants under artificial high light.

\section{Dynamics of anthocyanin accumulation and associated gene expression in Pro35S:BrTT8 seedlings under high- light treatments}

The simultaneous enhanced expression of the putative transcriptional activators SlAN2 and SITT8 and the repressor SIMYBL2 make it difficult to explain the underlying mechanisms of the profound production of anthocyanins in Pro35S:BrTT8 seedlings under high-light exposure. To obtain a better sense of how transcription factors coordinate the regulation of anthocyanin biosynthesis, the dynamics of anthocyanin accumulation and associated gene expression were analyzed. Pro35S:BrTT8 seedlings cultivated in low-light conditions were transferred to artificial high-light conditions for 7 days. Leaves were sampled from transgenic seedlings at consistent times after $0,12,36,72,120$ and $168 \mathrm{~h}$, and then total anthocyanin contents and the expression of anthocyanin biosynthetic and regulatory genes were examined. First, transcripts of BrTT8 were found to be stable in transgenic seedlings at different stages (Supplemental Figure 4). As shown in Fig. 4, anthocyanin production was absent in samples gathered at 0 and $12 \mathrm{~h}$, while it rose significantly at $36 \mathrm{~h}$ and reached its maximum value at $168 \mathrm{~h}$. Transcripts of all the structural genes increased swiftly just after the onset of high-light treatment and reached peak values within $72 \mathrm{~h}$. Thereafter, transcripts of all the structural genes except SIPAL, SlC4H and Sl4CL gradually declined to relatively stable levels, which were still drastically higher than the respective initial levels at 0 h (Fig. 4). These results show that anthocyanin accumulation lagged behind the transcriptional activation of structural genes in tomato seedlings upon high-light treatment.

As shown in Fig. 4, the transcripts of SlAN2 and SlTT8 increased drastically just after the onset of high-light treatment. In particular, transcripts of SlAN2 increased to their maximal values within $12 \mathrm{~h}$ and remained relatively stable at the remaining time points. However, transcripts of SITT8 increased slowly to their peak value and then displayed a declining trend. The dynamics of SITT8 transcripts resembled those of some anthocyanin structural genes mentioned above, suggesting that SlTT8 might be transcriptionally regulated in a similar manner at high-light exposure. However, the transcripts of SIMYBL2 remained relatively stable within the first $72 \mathrm{~h}$ but increased substantially near the end of the high-light treatment. Apparently, SIMYBL2 did not participate in the transcriptional regulation of anthocyanin structural genes at the earlier stage of the high-light treatment. In addition, the expression of the putative transcriptional repressor SITRY, belonging to R3-MYB family, seemed insensitive to high-light stress. 


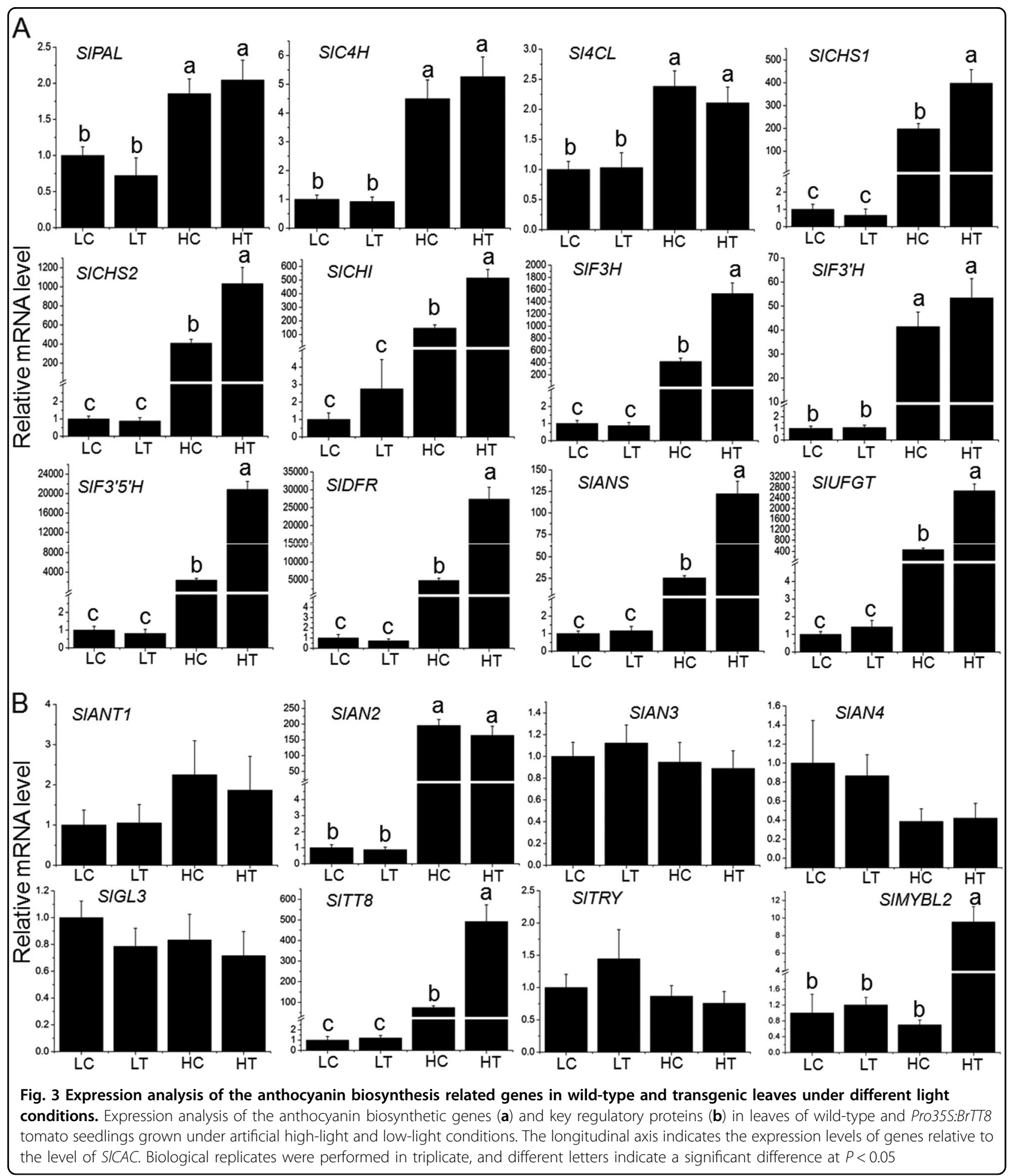

Nonuniform anthocyanin pigmentation in fruits of Pro35s: BrTT8 plants after exposure of natural high light

Visual inspection showed that anthocyanins accumulated specifically in roughly spherical areas of the upper epicarp around the stem end of Pro35S:BrTT8 fruits under natural high light, regardless of developmental stage (Fig. 5a, c). In addition, the total anthocyanin content of epicarps at the stem end of ripening fruits at the breaker stage was $0.26 \mathrm{mg} \mathrm{g}^{-1}$ dry weight, which was evidently lower than that of fruits at the green stage, suggesting that anthocyanins degrade notably during the fruit ripening process (Fig. 5e). However, no anthocyanins 


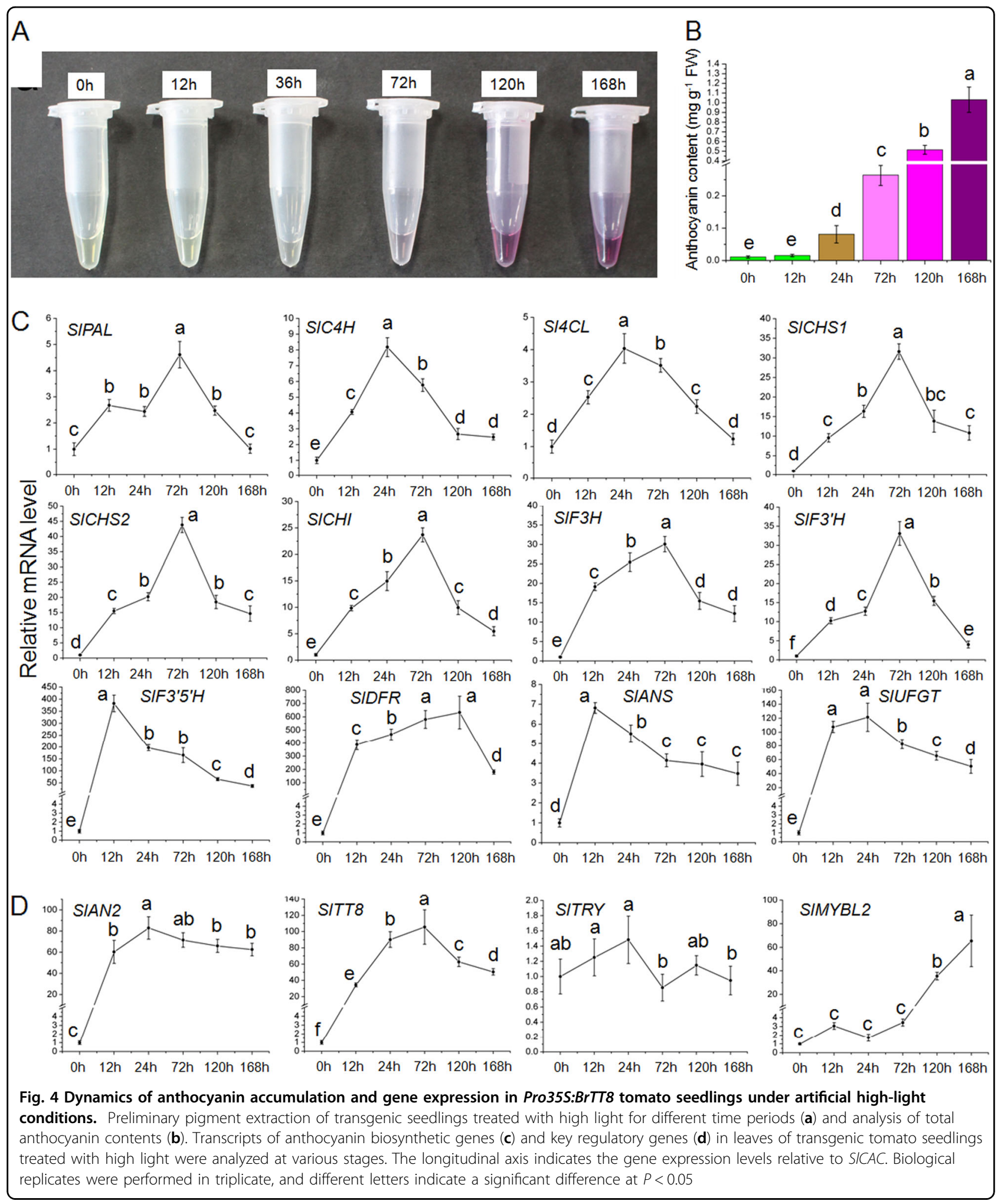

were detected in any fruit tissues of wild-type tomato plants under natural high-light conditions.

To investigate the molecular mechanisms underlying the region-specific anthocyanin pigmentation in tomato fruits of Pro35S:BrTT8 plants under natural high-light conditions, expression levels of the associated genes were analyzed in divided parts of wild-type and transgenic fruits as follows: EMW (epicarp at stem end of wild-type plants), MMW 

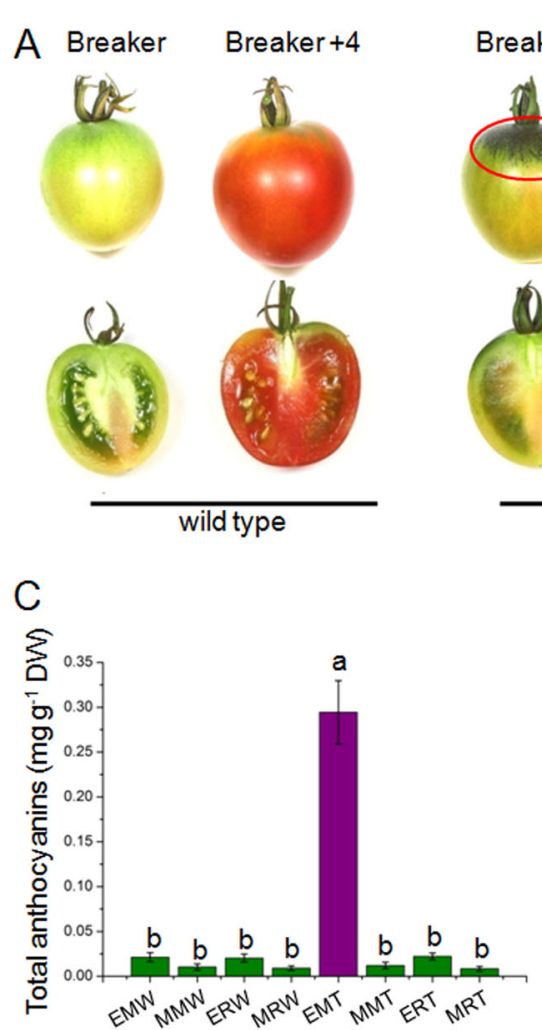
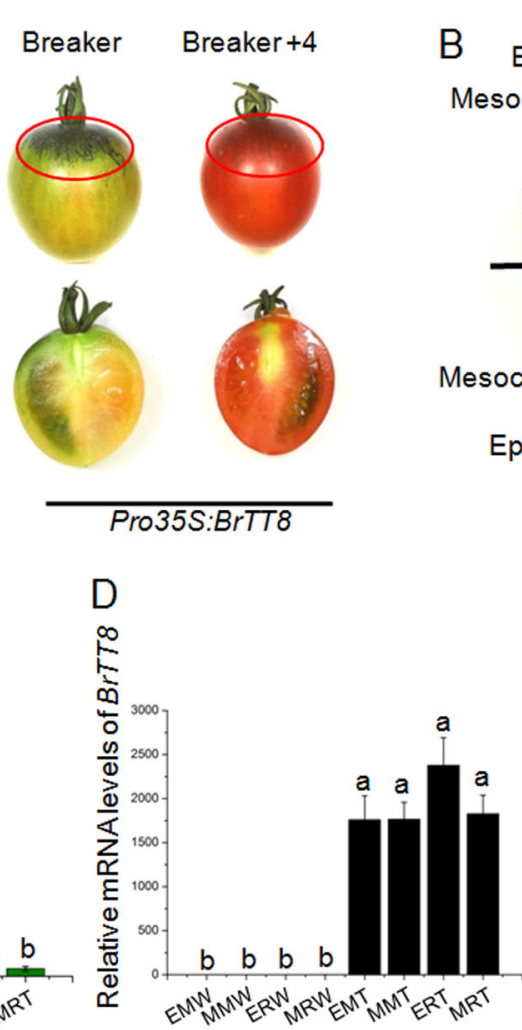
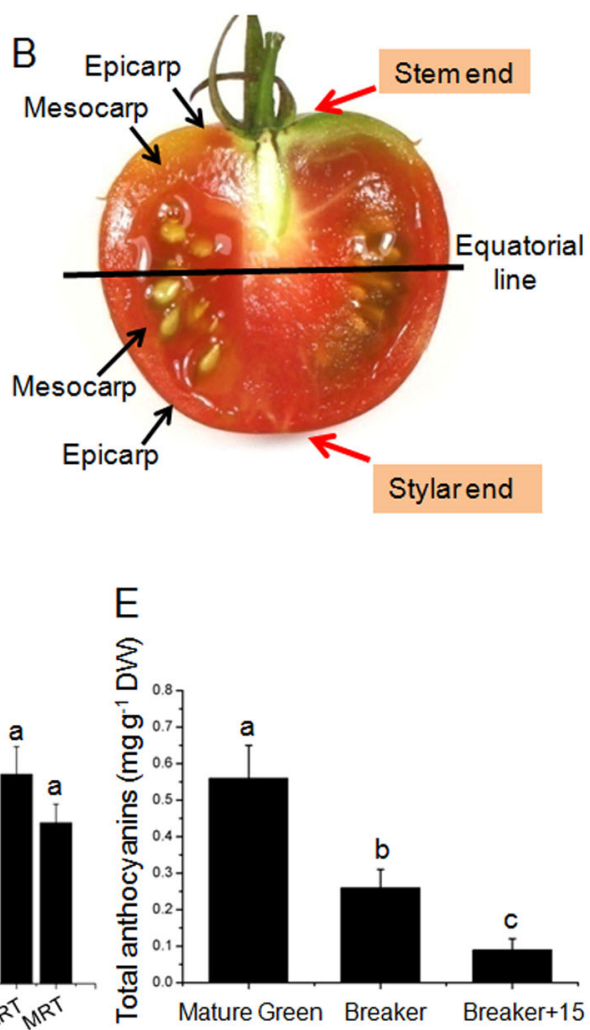

Fig. 5 Anthocyanins accumulated specifically in the upper epicarps of Pro35S:BrTT8 tomato plants grown under natural high-light conditions. a Regardless of the developmental stages, anthocyanins accumulated specifically in the upper epicarps of fruits under natural high-light conditions. b Schematic representation of the different parts of fruit used for pigment measurements and gene expression analysis. $\mathbf{c}$ Total anthocyanin contents in different parts of wild-type and Pro35S:BrTT8 tomato fruits at the Breaker + stage. $\mathbf{d}$ Expression analysis of BrTT8 in different fruit parts of wild-type and Pro35S:BrTT8 plants. The longitudinal axis indicates the expression level of BrTT8 relative to SICAC. e Total anthocyanin contents of epicarps in the stem end in Pro35S:BrTT8 tomato fruits at different development stages. Breaker +44 days after breaker, Breaker +15 15 days after breaker, EMW epicarp at the stem end of wild-type fruits, MMW mesocarp at the stem end of wild-type fruits, ERW epicarp at the stylar end of wild-type fruits, MRW mesocarp at the stylar end of wild-type fruits, EMT epicarp at the stem end of transgenic fruits, MMT mesocarp at the stem end of transgenic fruits, ERT epicarp at the stylar end of transgenic fruits, MRT mesocarp at the stylar end of transgenic fruits. Biological replicates were performed in triplicate, and different lowercase letters indicate significance at $P<0.05$

(mesocarp at stem end of wild-type plants), ERW (epicarp at stylar end of wild-type plants), MRW (mesocarp at stylar end of wild-type plants), EMT (epicarp at stem end of transgenic plants), MMT (mesocarp at stem end of transgenic plants), ERT (epicarp at stylar end of transgenic plants) and MRT (mesocarp at stylar end of transgenic plants) (Fig. 5b). Before this, BrTT8 transcripts were examined preferably and were consistently expressed in different regions of Pro35S:BrTT8 fruits (Fig. 5d). In fruits of wild-type plants, the expression levels of SlCHS1, SlCHS2, SlCHI, SIF3'H, SIF3' $5^{\prime} \mathrm{H}$, SIDFR and SIANS in epicarps at the stem end were significantly higher than those at the stylar end. Moreover, the expression levels of these genes were further enhanced on a large scale in epicarps at the stem end of Pro35S:BrTT8 fruits. However, only weak transcriptional signals for SlCHS1, SlCHS2, SlCHI, SlF3H, SlF3'H, SlF3'5'H, SIDFR, SlANS and SIUFGT were detected in mesocarps of wild-type plants at both the stem end and stylar end. In addition, these genes exhibited no evident differences in mesocarps of wild-type and Pro35S:BrTT8 plants (Fig. 6). These results provide a good explanation for the large amounts of flavonoid accumulation that occur in epicarps but not in mesocarps ${ }^{40}$.

In the wild-type epicarps, the transcripts of SlAN2 and SlTT8 in EMW were significantly higher than those in MMW, ERW, and MRW (Fig. 6). Similar expression patterns for SlAN2 and SITT8 were also found in Pro35S: BrTT8 fruits. Moreover, the expression levels of SITT8 and SIMYBL2 were further enhanced in EMT compared with EMW. Among the regulatory genes that showed increased transcripts in EMT, SITT8 was strongly upregulated (more than 20-fold) in comparison with EMW. Altogether, these results suggest that the region-specific expression of SIAN2, SITT8 and SIMYBL2 should be responsible for the unique pigmentation pattern in fruits of Pro35S:BrTT8 plants exposed to natural high light. 


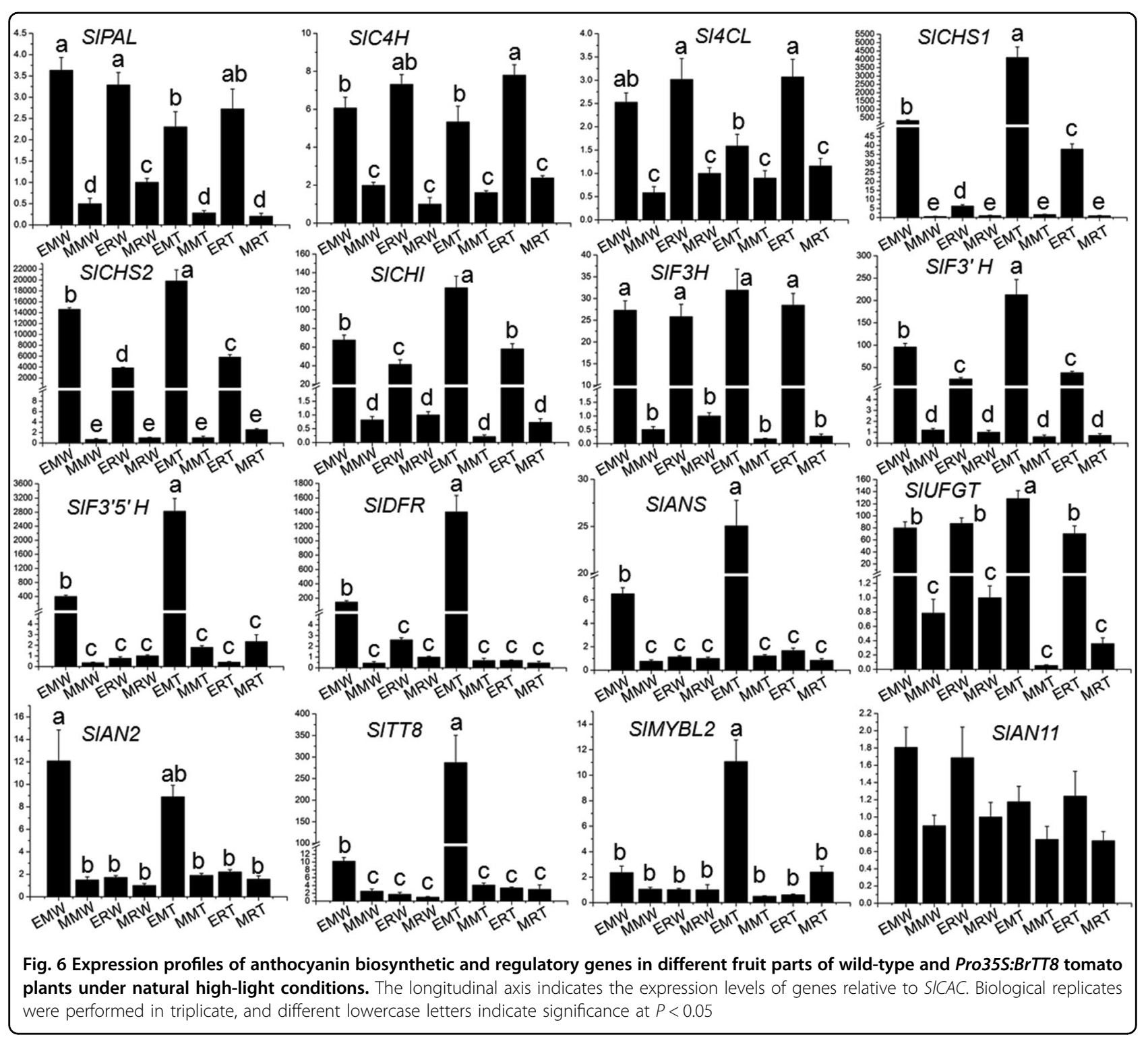

BrTT8 physically interacts with SIAN2, SIAN11, and SIMYBL2

Multiple sequence alignment of BrTT8, SITT8 and other bHLH anthocyanin regulators suggests that all the proteins share a typical bHLH domain and a Myb interaction region at the $\mathrm{N}$ terminus (Supplemental Figure S5). In addition, phylogenetic analysis of bHLH proteins from various plant species indicates that SITT8 is an ortholog of BrTT8, a functional anthocyanin activator (Supplemental Figure S6). Similarly, sequence analysis of MYB proteins from various species showed that SIAN2 and SIMYBL2 share the same conserved bHLH protein interaction motif with other transcriptional regulators (Supplemental Figures S7 and S8). To further unravel the molecular mechanism by which high light regulates anthocyanin accumulation in the leaves and fruits of
Pro35S:BrTT8 plants, Y2H assays were performed to identify candidate proteins that physically interact with BrTT8. Positive $\mathrm{X}-\mathrm{a}-\mathrm{Gal}$ activity was found in strains containing pGBKT7-BrTT8 \& pGADT7-SIAN2, pGBKT7-BrTT8 \& pGADT7-SIMYBL2 and pGBKT7BrTT8 \& pGADT7-SlAN11 grown on the QDO/X-a-Gal screening medium but not in those containing pGBKT7BrTT8 \& pGADT7-BrTT8 (Fig. 7a), indicating that BrTT8 interacts with SlAN2, SIMYBL2 and SlAN11 physically. An MYB-bHLH-WD40 protein complex that plays an essential regulatory role in anthocyanin biosynthesis has been studied extensively in previous reports $^{21,51}$. In addition, large amounts of transcripts of SlAN2, BrTT8, SlMYBL2 and SlAN11 were detected in tissues exhibiting abundant anthocyanin accumulation. Consequently, it is reasonable to speculate that Pro35S: 

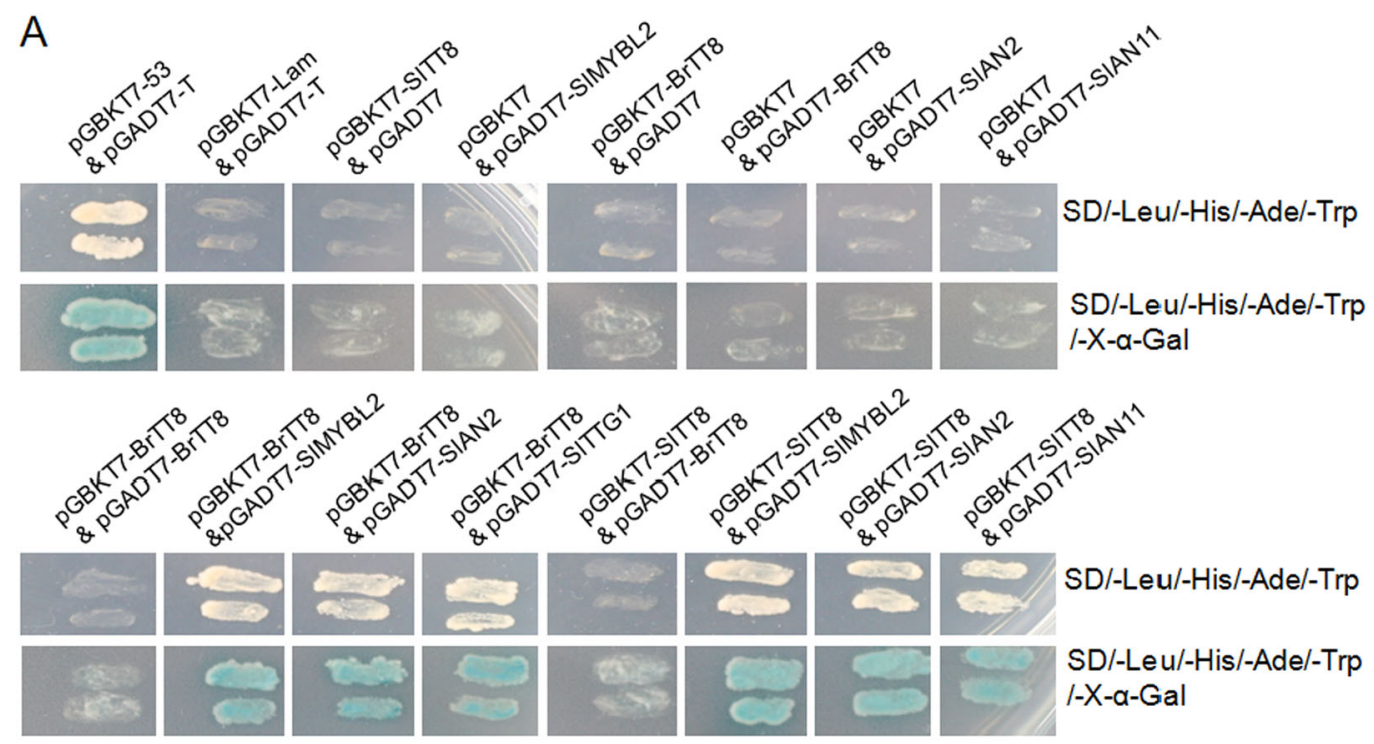

B

BrTT8-EGFP \& SIAN2-mCherry co-transfection FRET-APB
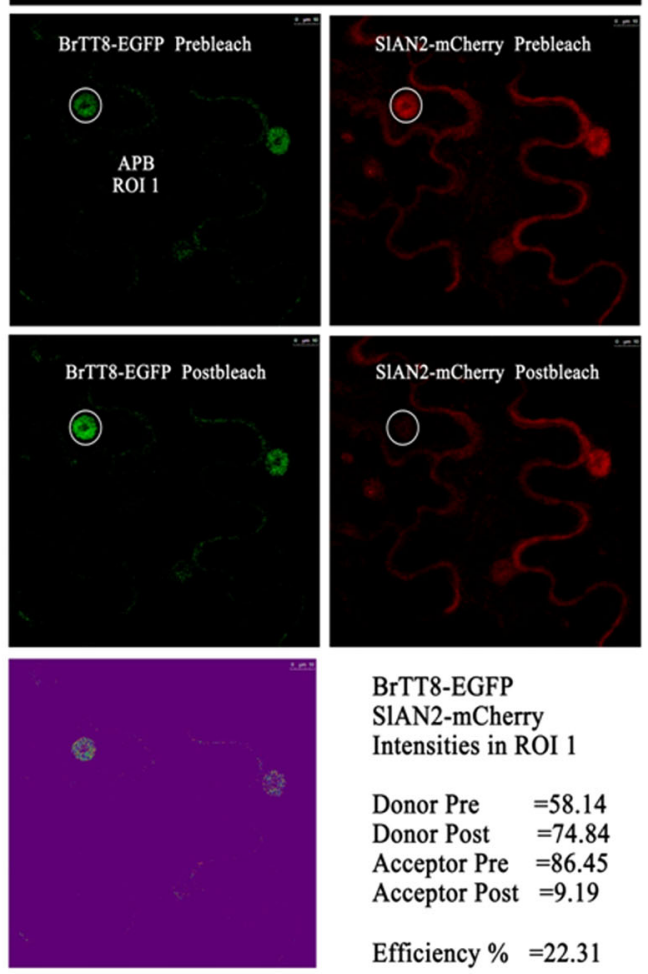

BrTT8-EGFP \& SIAN2-mCherry co-transfection FRET-APB

\begin{tabular}{c|c|c|c|c|} 
D Pre & D Post & A Pre & A Post & FRET(\%) \\
\hline 76.04 & 89.76 & 79.67 & 14.49 & 15.29 \\
\hline 60.79 & 75.14 & 94.23 & 10.26 & 19.10 \\
\hline 58.14 & 74.84 & 86.45 & 9.19 & 22.31 \\
\hline 69.95 & 85.16 & 84.78 & 15.45 & 17.86 \\
\hline & & Mean FRET & 18.64 \\
\cline { 5 - 5 }
\end{tabular}
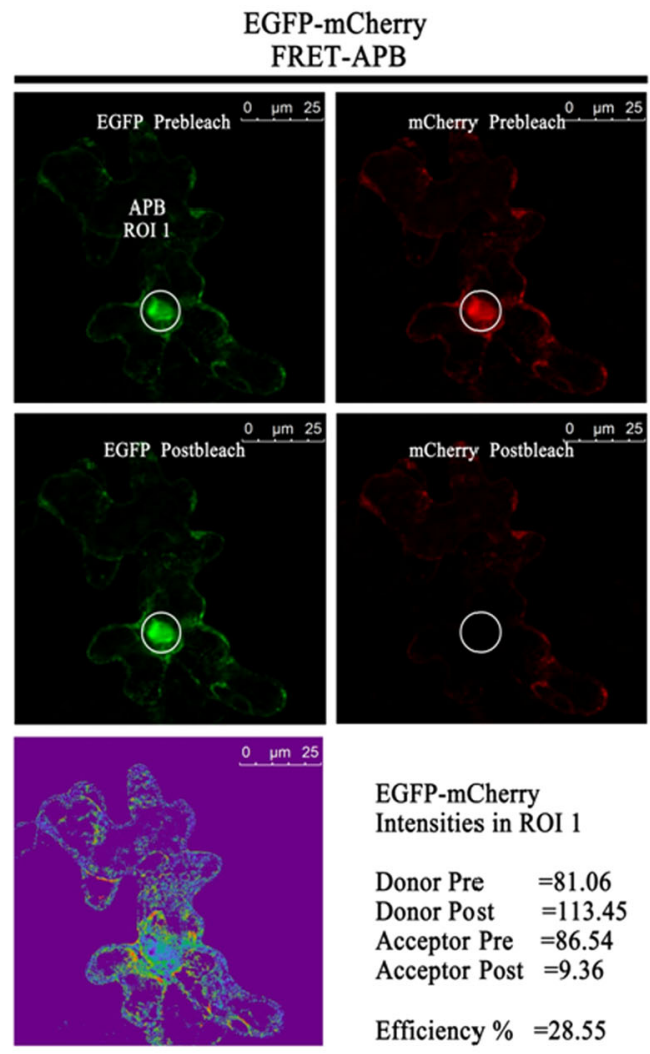

EGFP-mCherry

Intensities in ROI 1

Donor Pre $=81.06$

Donor Post $=113.45$

Acceptor Pre $=86.54$

Acceptor Post $=9.36$

Efficiency $\%=28.55$

\begin{tabular}{|c|c|c|c|c|}
\hline \multicolumn{5}{|c|}{$\begin{array}{c}\text { EGFP-mCherry } \\
\text { FRET-APB }\end{array}$} \\
\hline D Pre & D Post & A Pre & A Post & FRET(\%) \\
\hline 89.12 & 117.23 & 98.45 & 13.67 & 23.98 \\
\hline 79.90 & 101.93 & 104.44 & 10.57 & 21.61 \\
\hline 81.06 & 113.45 & 86.54 & 9.36 & 28.55 \\
\hline 80.34 & 102.24 & 82.28 & 16.40 & 21.42 \\
\hline & & & Mean FRET & 23.89 \\
\hline
\end{tabular}

Fig. 7 BrTT8 interacts physically with SIAN2, SIAN11, and SIMYBL2 in vivo. a Protein-protein interactions between members of the MBW regulatory complex were analyzed by yeast two-hybrid assays. b Physical interactions between BrTT8 and SIAN2 were further confirmed by fluorescence resonance energy transfer (FRET) 
BrTT8 plants adapt to high-light conditions by enhancing anthocyanin pigmentation in vegetative and fruit tissues via the transcriptional activity of a heterogeneous $\mathrm{MBW}$ complex consisting of BrTT8, SlAN2 and SlAN11. Additionally, the physical interaction between SIMYBL2 and BrTT8 indicates that SIMYBL2 might inhibit the assembly of the functional MYB-bHLH-WD40 protein complex by competitively binding with the MYB interaction domain in BrTT8. Positive X-a-Gal activity was also found in yeast strains containing pGBKT7-SITT8 \& pGADT7-SIAN2, pGBKT7-SITT8 \& pGADT7-SIMYBL2 and pGBKT7-SITT8 \& pGADT7-SIAN11 grown on QDO/X-a-Gal screening medium but not in those containing pGBKT7-SITT8 \& pGADT7-BrTT8 (Fig. 7a), suggesting that SITT8 could interact with the endogenous anthocyanin regulators physically but not with BrTT8. These results indicate that SITT8 might also participate in the assemblage of the endogenous MBW complex with SlAN2 and SlAN11. Thus, the MBW complex consisting of endogenous transcription factors SITT8, SlAN2 and SIAN11 might also contribute to anthocyanin accumulation. It seems that BrTT8 cannot form a homodimer with itself or a heterodimer with SITT8 in vivo. However, it is worth noting that the C-terminal regions of the BrTT8 and SlTT8 genes were deleted in $\mathrm{Y} 2 \mathrm{H}$ assays for the elimination of potential transcriptional activation activity.

To confirm the physical interaction between BrTT8 and SIAN2, the full-length coding sequences of BrTT8 and SlAN2 were fused with EGFP and mCherry protein, respectively. BrTT8 interacted with SIAN2 in the FRETAPB assays (Fig. 7b), confirming the presence of a heterogeneous MBW complex in Pro35S:BrTT8 tomato plants. Additionally, both BrTT8 and SIAN2 were located in the nucleus of agroinfiltrated tobacco epidermal cells visualized with laser scanning confocal microscopy. Altogether, these results support the hypothesis that a heterogeneous MBW complex consisting of BrTT8, SlAN2 and SlAN11 fundamentally regulates anthocyanin accumulation.

BrTT8, SIAN2 and SIMYBL2 coordinately regulate the biosynthesis of anthocyanins and polyphenols in tobacco

To further verify the hypothesis proposed above, expression vectors were generated following the schematic representation shown in Fig. 8a and then used for transient transformation of tobacco epidermal cells. Four days after infiltration, purple pigments emerged clearly in the epidermal tissues of tobacco leaves (Fig. 8b). Similar to the control, overexpression of SIMYBL2 did not result in the accumulation of purple pigments. Moreover, sole expression of BrTT8 failed to induce anthocyanin accumulation in infiltrated leaves, indicating inefficient interactions with the endogenous MYB and WD40 proteins or expression deficiency of endogenous MYB and WD40 proteins. In contrast, the ability of SlAN2 to induce anthocyanin accumulation, without coinfiltration of a bHLH protein, might be due to efficient interactions with the endogenous bHLH proteins in tobacco.

To further investigate the physical interactions between subunits of the MBW complex, different combinations of overexpression constructs were introduced into tobacco leaves by Agrobacterium tumefaciens-mediated transient expression. Among the infiltrated leaves, coinfiltration of BrTT8 and SlAN2 induced the highest amount of anthocyanin production; infiltration of SlAN2 induced the second highest; coinfiltration of BrTT8, SlAN2 and SIMYBL2 induced the third highest (Fig. 8b, d). In addition, delphinidin-3-O-runtinoside, as the main component of purple pigments, was extracted from all infiltrated patches and identified by HPLC-ESI-MS/MS (Fig. 8c), indicating that biosynthetic genes of the anthocyanin pathway were upregulated by the same mechanisms. Then, the transcript levels of anthocyanin biosynthetic and exogenous genes were examined in all the infiltrated patches (Supplemental Table S4 and Figure S9). Clearly, SIAN2, BrTT8 and endogenous WD40 protein coordinately promote anthocyanin pigmentation by activating the transcription of anthocyanin structural genes in tobacco cells, while SIMYBL2 prevents this transcriptional activation, probably by disturbing the formation of a functional MBW protein complex. Taking all these results into account, we can obtain three conclusions: (1) a functional MBW complex consisting of BrTT8, SIAN2, and endogenous WD40 protein is assembled efficiently in infiltrated tobacco leaves; (2) SIMYBL2 blocks high amounts of anthocyanin accumulation by preventing the assemblage of the functional MBW complex; and (3) the inability of BrTT8 to induce anthocyanin accumulation should be due to a deficiency in the expression of endogenous MYB proteins in tobacco.

Except for anthocyanins, the increased contents of phenolic acids and flavonols were also analyzed in infiltrated tobacco leaves (Table 2). The increased biosynthesis of neochlorogenic acid, chlorogenic acid, and cryptochlorogenic acid might be due to the upregulation of early anthocyanin biosynthetic genes, including $N b P A L$ and $\mathrm{NbC4H}$, since the biosynthesis of anthocyanin and phenolic acids share the same pathway in the initial steps of phenylpropanoid metabolism (Fig. 1). Similarly, it is possible that the enhanced biosynthesis of dihydroflavonol in the anthocyanin pathway contributes to the accumulation of kaempferol-3-O-rutinoside-7-O-glucoside (Table 2).

\section{BrTT8, SIAN2, and SIAN11 coordinately trigger anthocyanin production in tomato plants}

Together, a working model was proposed in which a heterogeneous MBW protein complex consisting of 


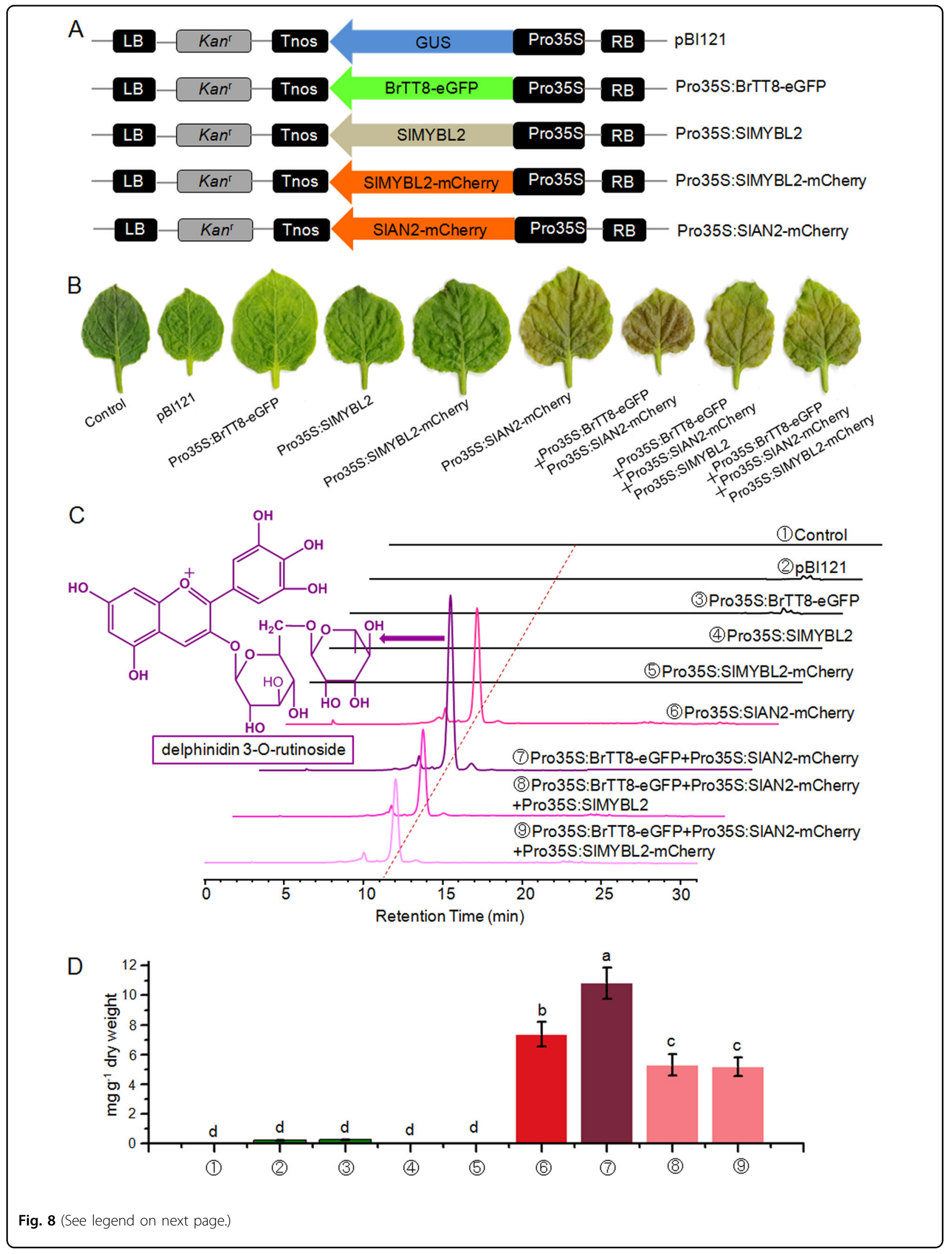


(see figure on previous page)

Fig. 8 SIAN2, BrTT8, and SIMYBL2 coordinately regulated the biosynthesis of anthocyanins in tobacco leaves. a Schematic representation of the overexpression constructs used for transformation of Nicotiana benthamiana leaves. $\mathbf{b}$ Anthocyanin pigmentation in Nicotiana benthamiana leaves 4 days after ectopic expression of different combinations of exogenous genes. c HPLC profiles of anthocyanins extracted from infiltrated patches 4 days after infiltration, and the structure of the main anthocyanin component identified by HPLC-ESI-MS/MS is shown in purple color. d Total anthocyanin contents of infiltrated patches in the control and leaves expressing different combinations of exogenous genes. Biological replicates were performed in triplicate, and different lowercase letters indicate significance at $P<0.05$

BrTT8, SlAN2, and SlAN11 triggers anthocyanin accumulation by increasing transcripts of biosynthetic genes in Pro35S:BrTT8 tomato plants grown under high-light conditions (Fig. 9). Furthermore, an endogenous MBW protein complex consisting of SITT8, SlAN2, and SIAN11 might also contribute to the transcriptional activation of anthocyanin biosynthesis in wild-type plants and enhance anthocyanin pigmentation further in Pro35S:BrTT8 tomato plants under high-light conditions. However, large amounts of anthocyanin accumulation in plant tissues certainly consumes considerable amounts of precursor phenylalanine and energy, leading to a clear growth delay to some extent ${ }^{52}$. Consequently, too much anthocyanin pigmentation might activate the expression of SIMYBL2 through unknown mechanisms to avoid excess anthocyanin accumulation in a negative-feedback manner.

\section{Nonuniform anthocyanin accumulation and gradient light flux received by epicarp cells in Pro35S:BrTT8 plants}

Fruits with uniform pigmentation always show better presentation and some tomato cultivars truly display uneven distribution of pigments in fruits ${ }^{53}$. The regionspecific anthocyanin pigmentation makes Pro35S:BrTT8 tomato a good model for metabolic and molecular studies of nonuniform fruit pigmentation in the plant kingdom. It was proven that light intensity has significant effects on anthocyanin accumulation in tomato plants in the earlier part of this article. As a result, the relationship between anthocyanin pigmentation patterns and direct high-light irradiance shed on epicarps of Pro35S:BrTT8 tomato plants was investigated.

As shown in Fig. 10, plant cells of mesocarps do not have the opportunity to absorb direct sunlight, and epicarps of the stylar end can only absorb scattered light (lower than $100 \mu \mathrm{mol}$ photons $\mathrm{m}^{-2} \mathrm{~s}^{-1}$ ), which is much lower than direct sunlight in light intensity. In fact, the actual direct light flux received by the epidermal cells in the unit area changes as the position of the spherical fruit surface moves (Fig. 10). As the upper surface of the tomato fruit is an approximate regular sphere, it is easy to estimate that the actual luminous flux received per unit area on the fruit epicarp was only equal to its projection area in the horizontal surface. Supposing that the value of luminous flux passing through a unit area at a unit time is A under natural high light, the value of luminous flux received in the same unit area of the epicarp was A multiplied by cosine $\theta$, where $\theta$ indicates the angle between a vertical line and a ligature connecting the unit area and sphere center (Fig. 10). Apparently, with the increase of the angle index, the value of cosine $\theta$ gradually decreases to zero, indicating a continuous reduction in the luminous flux of direct sunlight received in the unit area on the fruit surface. Consequently, the uneven flux of direct sunlight received by epidermal tissues provides a potential explanation for nonuniform anthocyanin pigmentation in tomato fruits upon exposure to natural sunlight. Moreover, the changing intensity of anthocyanin pigmentation in the upper epidermis is closely associated with the specific angle $\theta$, determined by a fixed position. No visible purple pigments were observed in the upper epidermal tissues near the equatorial line of fruit because the direct sunlight flux received by the corresponding tissues was very limited. These results indicated that the activation of anthocyanin biosynthesis requires a certain strength of direct high-light irradiance. In other words, once the direct high-light flux received by the cells on the epicarp is less than the minimum limit, the cells are unable to trigger anthocyanin biosynthesis.

\section{Low-light irradiance cannot trigger anthocyanin accumulation in the upper epicarp of Pro35S:BrTT8 tomato fruits}

To further verify the hypothesis proposed above, Pro35S:BrTT8 fruits were bagged with semitransparent bags immediately after pollination to simulate artificial low-light irradiance environments. Meanwhile, the whole plants were cultivated in a greenhouse under artificial high light. Compared with Pro35S:BrTT8 fruits exposed to artificial high light, no anthocyanins were detected in the upper epicarps of the bagged fruits (Fig. 11 and Supplemental Figure S10). The fruits exposed to artificial high light showed intense purple/black pigmentation in the shoulder region, and the total anthocyanin content was approximately $0.36 \mathrm{mg} \mathrm{g}^{-1} \mathrm{FW}$ in EMT, while the fruits covered with translucent plastic bags exhibited acyanic epicarps (Fig. 11 and Supplemental Figure S10). Transcripts of most anthocyanin structural genes, including CHS1, CHS2, CHI, F3H, F3' H, F3' $5^{\prime} H$, DFR and ANS, of bagged EMTs were drastically less than those of EMTs exposed to high light (Fig. 11e). In addition, the low 


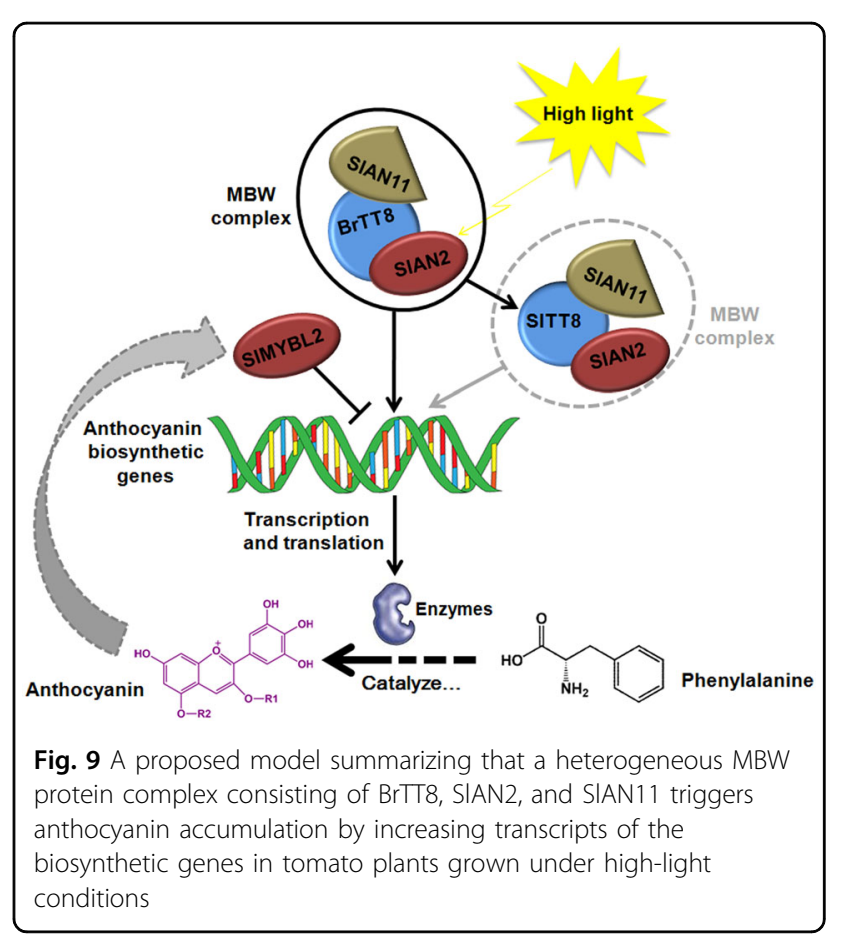

expression levels of anthocyanin activators, SlAN2 and SITT8, further prove the inability of low-light irradiance to transcriptionally activate the expression of anthocyanin structural genes. Based on these results, we conclude that nonuniform anthocyanin pigmentation in Pro35S:BrTT8 fruit epicarps is due to temporal and spatial expression of SlAN2 triggered by uneven high-light irradiance received by epidermal tissues. In addition, chlorophyll biosynthesis was clearly inhibited in fruits under artificial low-light conditions compared with fruits exposed to high light (Supplemental Figure S10).

\section{Discussion}

In this study, Pro35S:BrTT8 tomato plants were utilized to study light intensity effects on anthocyanin pigmentation, especially in reproductive tissues. High light could effectively trigger anthocyanin accumulation in both the leaves and fruits of Pro35S:BrTT8 tomato plants. Moreover, anthocyanin pigmentation was uneven on the fruit surface, and the nonuniform distribution pattern was attributed to the direct sunlight flux received by epicarp tissue in the unit area of the fruit surface. Only in the epicarp cells that received a sufficient amount of light irradiance was the expression of the anthocyanin biosynthesis regulatory genes triggered, subsequently leading to enhanced expression of the anthocyanin biosynthetic genes and production of anthocyanins. Furthermore, it was demonstrated that anthocyanin pigmentation in both vegetative and reproductive tissues is mainly triggered by a heterogeneous MBW protein complex consisting of BrTT8, SlAN2 and SlAN11. Moreover, an R2R3-MYB 


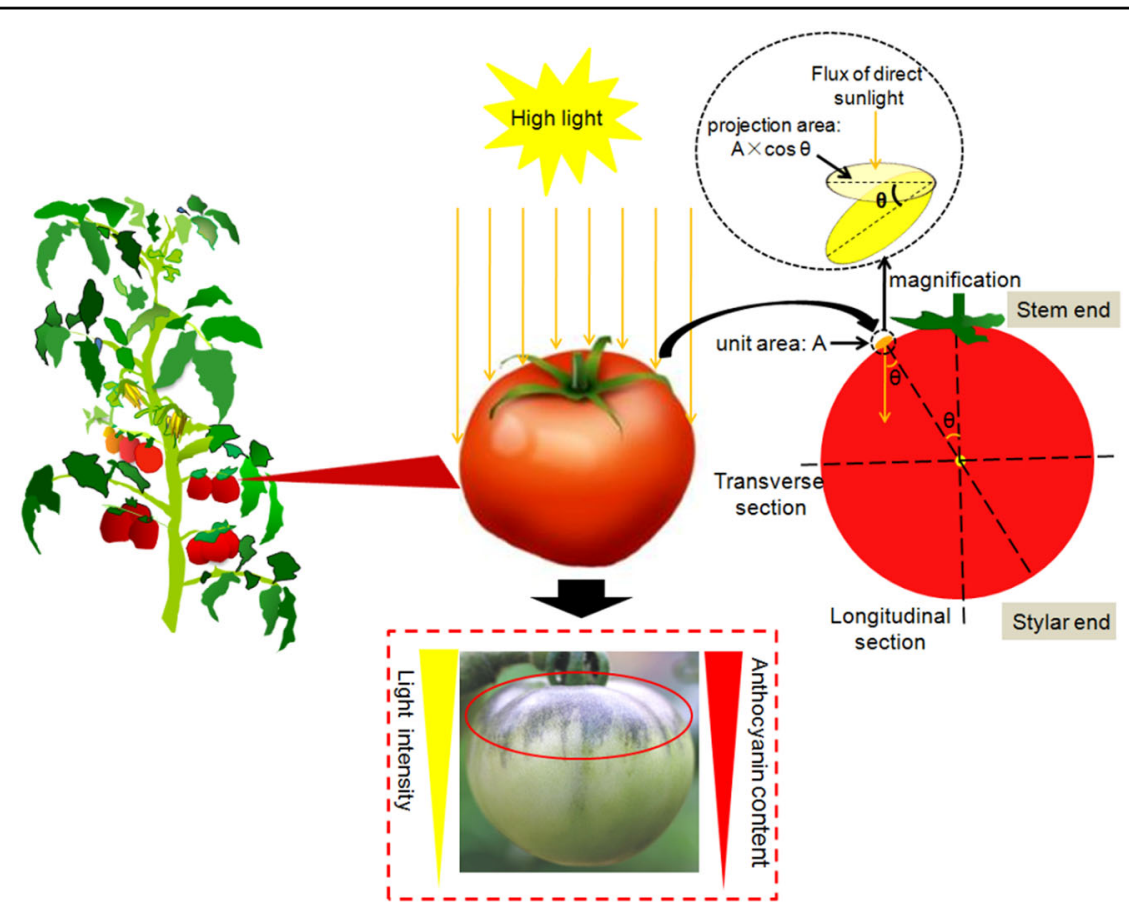

Fig. 10 Schematic representation of nonuniform anthocyanin distribution and direct sunlight received by epicarp tissues of Pro35S:BrTा8 tomato fruits under natural high-light conditions

repressor (SIMYBL2) can suppress the assemblage of the functional MBW complex by competitively binding with BrTT8, leading to the restricted production of anthocyanins. An excessive amount of anthocyanin production may serve as a signaling molecule to activate the expression of SIMYBL2, a competitive inhibitor of SlAN2, to maintain the balance between primary metabolism and secondary metabolism. To summarize all the findings in this study, a model that expands our understanding of the nonuniform anthocyanin pigmentation in tomato fruits under natural high-light conditions was proposed in Figs. 9 and 10.

\section{High-light-induced anthocyanin pigmentation in vegetative tissues of Pro35S:BrTT8 plants was triggered by SIAN2}

The light-induced expression of $B r T T 8$ was responsible for the intense purple pigments in bok choy ${ }^{45}$, and large amounts of anthocyanin pigmentation were only detected in Pro35S:BrTT8 plants under high-light conditions. Meanwhile, anthocyanin pigmentation was observed mainly in leaf veins at a modest level in wild-type plants (Fig. $2 \mathrm{e}-\mathrm{i}$ ). In contrast, all tomato plants displayed an anthocyaninless phenotype under low-light conditions, regardless of their genetic background. Together, these results prove that light intensity has vital effects on anthocyanin accumulation in vegetative tissues of Pro35S: BrTT8 tomato plants, according to the findings reported in transgenic petunia ectopically expressing Lc, a bHLH transcription factor that is homologous to BrTT $8^{35}$. These results strongly suggest that the bHLH transgene alone is insufficient to induce anthocyanin biosynthesis effectively under low-light conditions. Therefore, it is reasonable to assume that BrTT8 may act with endogenous proteins induced by high light to initiate anthocyanin biosynthesis in Pro35S:BrTT8 tomato plants. Therefore, both reported and putative direct regulators of anthocyanin biosynthesis were analyzed in the LC, HC, LT, and HT samples carefully. Most endogenous MYB regulators, including SlANT1, SlAN3 and SlAN4, SlAN11 (WD40 protein), SIGL3 (bHLH protein) and SITRY (R3-MYB) were shown to be insensitive to changes in light intensity (Fig. 3b). The significant upregulation of SIAN2, SITT8 and SIMYBL2 in both wild-type and Pro35S:BrTT8 seedlings aroused our attention. In particular, the further enhanced expression of SITT8 in transgenic seedlings under high light indicates that it may be coordinately regulated by BrTT 8 and other endogenous regulators (Fig. 3b), suggesting SITT8 should not be a direct candidate for the regulator receiving transduction signals from high light.

Sequence analysis of MYB proteins suggests that SIAN2 may serve as a functional anthocyanin activator (Supplementary Figure S7 and S8). In addition, $\mathrm{Y} 2 \mathrm{H}$ assays showed that BrTT8 could interact physically with endogenous regulators, including SIAN2, SIMYBL2 and SlAN11 (Fig. 7). Extensive studies have shown that 


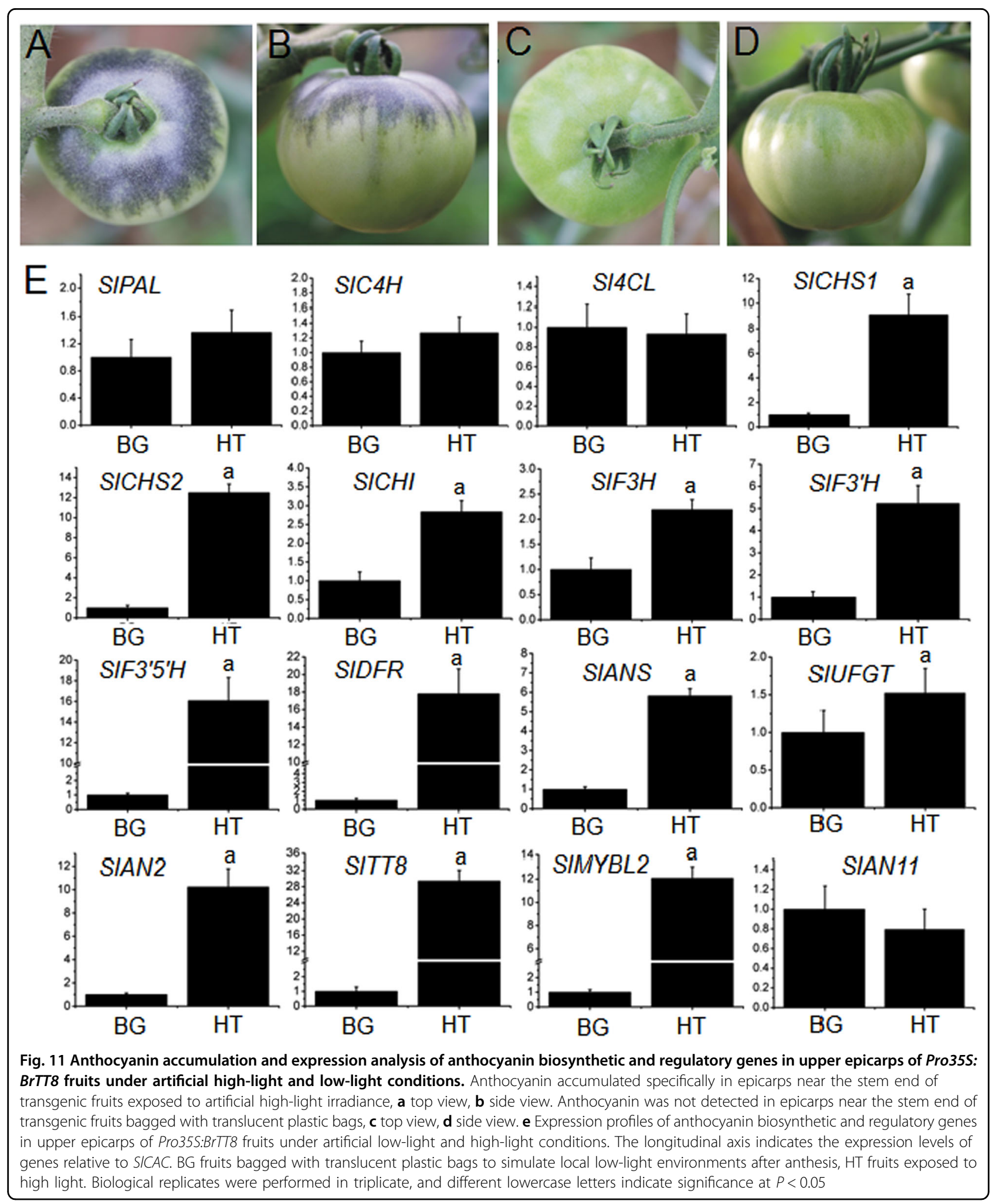

anthocyanin biosynthetic genes are regulated directly by the MBW complex consisting of MYB, bHLH and WDR proteins ${ }^{51,54-56}$. Therefore, it was supposed that high- light-induced anthocyanin pigmentation in Pro35S:BrTT8 seedlings was triggered by a heterogeneous $\mathrm{MBW}$ protein complex consisting of BrTT8, SlAN2, and SlAN11 
(Fig. 9). As noteworthy production of anthocyanins also occurred in wild-type plants, an endogenous MBW protein complex consisting of endogenous transcription factors SIAN2, SITT8, and SIAN11 may also contribute to the transcriptional activation of biosynthetic genes under high light. Moreover, the endogenous MBW protein complex might enhance anthocyanin pigmentation further in Pro35S:BrTT8 tomato plants under high-light conditions. SIAN2 was demonstrated to have the ability to induce the biosynthesis of anthocyanins, phenolic acids and flavonols in infiltrated tobacco leaves, and BrTT8 could enhance the function of SlAN2. In contrast, SIMYBL2 significantly inhibited the accumulation of these secondary metabolites.

With the extensive evidence shown above, it was demonstrated that a heterogeneous MBW protein complex consisting of BrTT8, SlAN11 and SIAN2 triggered anthocyanin pigmentation in vegetative tissues of transgenic tomato plants under high light by transcriptional activation of structural genes (Fig. 9). Together with further enhanced expression of SlTT8 in Pro35S:BrTT8 plants exposed to high light, we may infer that SlTT8 might be directly upregulated by the newly assembled heterogeneous MBW protein complex (Fig. 9). Moreover, the upregulated SITT8 might interact physically with the endogenous regulators SlAN11 and SlAN2, resulting in the formation of an endogenous MBW complex (marked with a dashed cycle) and further enhanced anthocyanin production in Pro35S:BrTT8 plants.

Sequence analysis showed that the $[\mathrm{R} / \mathrm{K}] \mathrm{Px}[\mathrm{P} / \mathrm{A} / \mathrm{R}] \mathrm{xx}[\mathrm{F} /$ $\mathrm{Y}]$ motif conserved in MYB anthocyanin activators was absent from the $\mathrm{C}$ terminus of SIMYBL2 ${ }^{57}$, in spite of an entire R2 domain at its $\mathrm{N}$ terminal region (Supplemental Figure S7). In addition, SIMYBL2 interacted with BrTT8 effectively in the $\mathrm{Y} 2 \mathrm{H}$ assay. These results indicate that SIMYBL2 inhibits anthocyanin biosynthesis by competitively binding with the MYB interaction domain of BrTT8, preventing the formation of a functional MBW complex (Fig. 9). The dynamics of anthocyanin production and gene expression in Pro35S:BrTT8 plants under high-light treatment prove that SIMYBL2 did not participate in the transcriptional regulation of anthocyanin structural genes in the early stages until the pigmentation intensity became high. Since too much anthocyanin accumulation can act as sunscreen to prevent light from being absorbed by chlorophyll pigments in leaves ${ }^{35}$, the induction of SIMYBL2 probably counterbalanced the high transcriptional activity of the MBW complex to avoid excessive anthocyanin production in a negative-feedback manner (Fig. 9). Since PhMYB27, an orthologous gene of SIMYBL2, was identified as a direct target of AN1 in petunia petals ${ }^{26}$, it can be speculated that SIMYBL2 might be transcription activated by the MBW complex itself under certain environments.

\section{Nonuniform anthocyanin pigmentation in fruits of Pro35s: BrTT8 tomato plants triggered by region-specific expression of SIAN2 under high light}

Delphinidin-based anthocyanins were identified as the main components in Pro35S:BrTT8 tomato fruits (Table 1 and Supplemental Figure S1). In the flavonoid biosynthetic pathway, $\mathrm{F}^{\prime} 5^{\prime} \mathrm{H}$ catalyzed the conversion of DHK to DHM by adding hydroxyl groups to the $3^{\prime}$ and $5^{\prime}$ positions on the $\mathrm{B}$ ring, leading to the synthesis of delphinidin-based anthocyanins (Fig. 1). Therefore, the drastic upregulation of $\mathrm{SmF}^{\prime} 5^{\prime} \mathrm{H}$ was responsible for the abundant accumulation of delphinidin-3-rutinoside-5-glucoside in the epicarps of Pro35S:BrTT8 plants. Furthermore, all anthocyanins identified in Pro35S:BrTT8 fruits were glycosylated anthocyanidins that arise from glycosylation at the $\mathrm{C} 5$ and $\mathrm{C} 3$ positions of the core skeleton. From another perspective, these results prove that glycosylation was the main type of modification for anthocyanin precursors found in Pro35S:BrTT8 tomato fruits.

Unlike vegetative tissues, anthocyanin pigmentation in Pro35S:BrTT8 fruit exposed to natural high light was restricted in an approximately spherical area around the stem end. To further elucidate the unique pattern of anthocyanin accumulation, fruits were divided into four parts in wild-type and transgenic plants (Fig. 5b). The expression profiles of anthocyanin structural genes matched well with the pigment distribution patterns in fruits (Figs. 5c and 6). Apparently, the unique pattern of nonuniform anthocyanin pigmentation in fruits of Pro35S: BrTT8 plants arises from region-specific expression of SIAN2 induced by natural high light. Similarly, the repressor SIMYBL2 was transcriptionally activated to prevent the excess production of anthocyanins. Herein, it seems that the molecular mechanisms underlying vegetative anthocyanin pigmentation are also suited for the coloration of Pro35S:BrTT8 fruits under natural high light. However, it is not clear whether region-specific expression of SIAN2 arises from tissue specificity or highlight irradiance.

The results shown in Fig. 11 proved that intense purple pigmentation of the upper epicarps can also be induced efficiently by artificial high light. Moreover, transcripts of anthocyanin biosynthetic and regulatory genes in the upper epicarps of Pro35S:BrTT8 fruits under both artificial low-light and high-light conditions were examined. It is possible that high light induced the high expression of SlAN2 in the upper epicarps, leading to the abundant assemblage of the MBW protein complex with BrTT8 and SlAN11 as cofactors. Ultimately, the region-specific enrichment of biosynthetic genes regulated by the MBW complex directly results in the unique distribution of anthocyanins in Pro35S:BrTT8 fruits exposed to natural high light. However, even in the upper part of the epicarps, the pigment distribution was not uniform. To study 
this biological phenomenon thoroughly, a model describing the actual dose of light flux received by the fruit surface (epicarp tissue) under high light was proposed (Fig. 10). Generally, the equatorial lines of most fruits are parallel to the horizontal surface, and natural sunlight is approximately vertical to the surface. As the model shows, the actual value of luminous flux received in a unit area on epidermal tissue was only equal to the cosine $\theta$ proportion of direct sunlight passing through the same area on the horizontal surface. Therefore, the actual direct sunlight flux received in the unit area of epicarps decreased sharply with the enlargement of the angle $\theta$. As a result, we speculate that light intensity below a certain value is insufficient to trigger visible anthocyanin production. As the unit area of the epicarps approaches the equatorial line, the actual dose of direct light received approaches zero, failing to induce visible anthocyanin accumulation. Logically, the pattern of direct sunlight intensity shed on the fruit surface coordinated well with the anthocyanin pigmentation strength. However, this model needs to be refined in future studies. Similarly, it was also reported that the green shoulder in AC results from a gradient pattern of expression of the plastid development regulator GLK2 throughout the fruit ${ }^{53}$. However, transcriptome profiling analysis revealed a broader gene expression gradient throughout the development of tomato fruits and rice leaves ${ }^{53,58}$. In terms of tissue patterning, several axes of developmental and morphological variation were proposed in fruit: apicalbasal, medial-lateral, and abaxial-adaxial ${ }^{59}$. However, it is difficult to verify whether the gradient expression pattern of these genes arises from a gradient of tissue specificity from the stem end to the stylar end or from environmental factors. Altogether, the gradient of the sunlight flux received by epidermal cells at different latitudes on the fruit surface was responsible for the unique pattern of anthocyanin distribution in Pro35S:BrTT8 epicarps exposed to natural high light.

Several positive regulators (SlAN2, SIANT1 and SlAN11) of anthocyanin accumulation in tomato plants have been characterized and investigated in previous studies $^{25,33,60,61}$. However, the negative regulator ATV encodes an R3-MYB protein that can directly bind bHLH proteins and acts as a competitive inhibitor ${ }^{33}$. Recently, even the crosstalk between ethylene signal transduction and anthocyanin biosynthesis was stu$\operatorname{died}^{52}$. However, nonuniform tomato fruit pigmentation under high light has not been studied. Remarkably, this article provides a good example of anthocyanin biosynthesis analysis in tomato fruits at metabolic and molecular levels under different light intensities. Furthermore, a model interpreting the nonuniform anthocyanin pigmentation of tomato fruits under natural high-light conditions was proposed. This article provides more insights into the biosynthesis and accumulation of nutritional substances in special environments and the genetic improvement of the market value of fruits and vegetables with future breeding.

\section{Acknowledgements}

We thank Dr. Jia Guo and Fangjie Xiong for valuable discussions. This work was supported by the National Natural Science Foundation of China (nos. 31601760, 31301778) and the Industry-University-Research Collaboration Grants of Henan Province (172107000020).

\section{Authors' contributions}

Y.Z., Y.L., Z.H., Y.T., W.L., J.H. and X.Y. conceived of the experiments. Y.L. and G.C. conducted the experimentation and analysis of data, with the following exceptions: X.Y. generated the transgenic tomato plants; J.H. performed the HPLC-ESI-MS/MS assays; W.L. performed the FRET analysis; Y.T. performed the total anthocyanin content analysis; M.Z. performed the high-light treatment; and Z.H. performed and analyzed Y2H experiments. Y.Z. and G.C. wrote the article, with assistance from all coauthors.

\section{Conflict of interest}

The authors declare that they have no conflict of interest.

\section{Publisher's note}

Springer Nature remains neutral with regard to jurisdictional claims in published maps and institutional affiliations.

Supplementary Information accompanies this paper at (https://doi.org/ 10.1038/s41438-019-0138-2).

Received: 18 September 2018 Revised: 7 February 2019 Accepted: 13 February 2019

Published online: 01 June 2019

\section{References}

1. Bradshaw, H. D. \& Schemske, D. W. Allele substitution at a flower colour locus produces a pollinator shift in monkeyflowers. Nature 426, 176-178 (2003).

2. Koes, R., Verweii, W. \& Quattrocchio, F. Flavonoids: a colorful model for the regulation and evolution of biochemical pathways. Trends Plant Sci. 10, 236-242 (2005).

3. Huang, J. et al. Comparative transcriptome analysis of the skin-specific accumulation of anthocyanins in black peanut (Arachis hypogaea L.). J. Agric. Food Chem. 67, 1312-1324 (2019).

4. Hichri, I. et al. Recent advances in the transcriptional regulation of the flavonoid biosynthetic pathway. J. Exp. Bot. 62, 2465-2483 (2011).

5. Hannum, S. M. Potential impact of strawberries on human health: a review of the science. Crit. Rev. Food Sci. Nutr. 44, 1-17 (2004).

6. Guarnieri, S., Riso, P. \& Porrini, M. Orange juice vs vitamin C: effect on hydrogen peroxide-induced DNA damage in mononuclear blood cells. Br. J. Nutr. 97, 639-643 (2007).

7. Shahidi, F. \& Ambigaipalan, P. Phenolics and polyphenolics in foods, beverages and spices: antioxidant activity and health effects-a review. J. Funct. Foods 18, 820-897 (2015).

8. Chun, O. K., Kim, D.-O. \& Lee, C. Y. Superoxide radical scavenging activity of the major polyphenols in fresh plums. J. Agric. Food Chem. 51, 8067-8072 (2003).

9. Butelli, E. et al. Enrichment of tomato fruit with health-promoting anthocyanins by expression of select transcription factors. Nat. Biotechnol. 26, 1301-1308 (2008).

10. Qin, Y. et al. Cyanidin-3-O-glucoside ameliorates diabetic nephropathy through regulation of glutathione pool. Biomed. Pharmacother. 103, 1223-1230 (2018).

11. Zhu, F. Anthocyanins in cereals: composition and health effects. Food Res. Int. 109, 232-249 (2018).

12. Vogt, T. Phenylpropanoid biosynthesis. Mol. Plant 3, 2-20 (2010). 
13. Sun, Y., Li, H. \& Huang, J.-R. Arabidopsis TT19 functions as a carrier to transport anthocyanin from the cytosol to tonoplasts. Mol. Plant 5, 387-400 (2012).

14. Luo, H. et al. Reduced Anthocyanins in Petioles codes for a GST anthocyanin transporter that is essential for the foliage and fruit coloration in strawberry. J. Exp. Bot. 69, 2595-2608 (2018).

15. Docimo, T. et al. Phenylpropanoids accumulation in eggplant fruit: characterization of biosynthetic genes and regulation by a MYB transcription factor. Front. Plant Sci. 6, 1233 (2015).

16. Outchkourov, N. S. et al. Transcription factor-mediated control of anthocyanin biosynthesis in vegetative tissues. Plant Physiol. 176, 1862-1878 (2018).

17. Feller, A., Machemer, K., Braun, E. L. \& Grotewold, E. Evolutionary and comparative analysis of MYB and bHLH plant transcription factors. Plant J. 66 , 94-116 (2011).

18. Schwinn, K. et al. A small family of MYB-regulatory genes controls floral pigmentation intensity and patterning in the genus Antirrhinum. Plant Cell $\mathbf{1 8}$ 831-851 (2006).

19. Stracke, R. et al. Differential regulation of closely related R2R3-MYB transcription factors controls flavonol accumulation in different parts of the Arabidopsis thaliana seedling. Plant J. 50, 660-677 (2007).

20. Lalusin, A. G., Nishita, K., Kim, S. H., Ohta, M. \& Fujimura, T. A new MADS-box gene (IbMADS10) from sweet potato (Ipomoea batatas (L.) Lam) is involved in the accumulation of anthocyanin. Mol. Genet. Genom. 275, 44-54 (2006).

21. Gonzalez, A., Zhao, M., Leavitt, J. M. \& Lloyd, A. M. Regulation of the anthocyanin biosynthetic pathway by the TTG1/bHLH/Myb transcriptional complex in Arabidopsis seedlings. Plant J. 53, 814-827 (2008).

22. Schwinn, K. E. et al. MYB and bHLH transcription factor transgenes increase anthocyanin pigmentation in petunia and lisianthus plants, and the petunia phenotypes are strongly enhanced under field conditions. Front. Plant Sci. 5, 603 (2014).

23. Liu, C. C. et al. The bZip transcription factor HY5 mediates CRY1ainduced anthocyanin biosynthesis in tomato. Plant Cell Environ. 41, 1762-1775 (2018).

24. Xu, W., Dubos, C. \& Lepiniec, L. Transcriptional control of flavonoid biosynthesis by MYB-bHLH-WDR complexes. Trends Plant. Sci. 20, 176-185 (2015).

25. Gao, Y. et al. Tomato SIAN11 regulates flavonoid biosynthesis and seed dormancy by interaction with bHLH proteins but not with MYB proteins. Hortic. Res. 5, 27 (2018)

26. Albert, N. W. et al. Members of an R2R3-MYB transcription factor family in Petunia are developmentally and environmentally regulated to control complex floral and vegetative pigmentation patterning. Plant J. $65,771-784$ (2011).

27. Jin, H. et al. Transcriptional repression by AtMYB4 controls production of UVprotecting sunscreens in Arabidopsis. EMBO J. 19, 6150-6161 (2000).

28. Aharoni, A. et al. The strawberry FaMYB1 transcription factor suppresses anthocyanin and flavonol accumulation in transgenic tobacco. Plant J. $\mathbf{2 8}$ 319-332 (2001).

29. Zhu, H. F., Fitzsimmons, K., Khandelwal, A. \& Kranz, R. G. CPC, a single-repeat R3 $M Y B$, is a negative regulator of anthocyanin biosynthesis in Arabidopsis. Mol. Plant 2, 790-802 (2009).

30. Nukumizu, Y., Wada, T. \& Tominaga-Wada, R. Tomato (Solanum lycopersicum) homologs of TRIPTYCHON (SITRY) and GLABRA3 (SIGL3) are involved in anthocyanin accumulation. Plant Signal. Behav. 8, e24575 (2013)

31. Wada, T., Kunihiro, A. \& Tominaga-Wada, R. Arabidopsis CAPRICE (MYB) and GLABRA3 (bHLH) control tomato (Solanum lycopersicum) anthocyanin biosynthesis. PLOS ONE 9, e109093 (2014).

32. Cao, X. et al. A putative R3 MYB repressor is the candidate gene underlying atroviolacium, a locus for anthocyanin pigmentation in tomato fruit. J. Exp. Bot. 68, 5745-5758 (2017).

33. Colanero, S., Perata, P. \& Gonzali, S. The atroviolacea gene encodes an R3-MYB protein repressing anthocyanin synthesis in tomato plants. Front. Plant Sci. $\mathbf{9}$, 830 (2018).

34. Merzlyak, M. N., Chivkunova, O. B., Solovchenko, A. E. \& Naqvi, K. R. Light absorption by anthocyanins in juvenile, stressed, and senescing leaves. J. Exp. Bot. 59, 3903-3911 (2008).

35. Albert, N. W. et al. Light-induced vegetative anthocyanin pigmentation in Petunia. J. Exp. Bot. 60, 2191-2202 (2009).

36. Lei, $\mathbf{M}$. et al. Genetic and genomic evidence that sucrose is a global regulator of plant responses to phosphate starvation in Arabidopsis. Plant Physiol. 156 1116-1130 (2011).
37. Xie, X. B. et al. The bHLH transcription factor MdbHLH3 promotes anthocyanin accumulation and fruit colouration in response to low temperature in apples. Plant Cell Environ. 35, 1884-1897 (2012).

38. Lloyd, A. M., Walbot, V. \& Davis, R. W. Arabidopsis and Nicotiana anthocyanin production activated by maize regulators R and C1. Science 258, 1773 (1992).

39. Mark, M. et al. Altered regulation of tomato and tobacco pigmentation genes caused by the delila gene of Antirrhinum. Plant J. 7, 333-339 (1995).

40. Bovy, A. High-flavonol tomatoes resulting from the heterologous expression of the maize transcription factor genes LC and C1. Plant Cell Online 14, 2509-2526 (2002).

41. Qiu, Z. et al. The Tomato Hoffman's anthocyaninless gene encodes a bHLH transcription factor involved in anthocyanin biosynthesis that is developmentally regulated and induced by low temperatures. PLOS ONE 11, e0151067 (2016).

42. Kiferle, C. et al. Tomato R2R3-MYB proteins SIANT1 and SIAN2: same protein activity, different roles. PLOS ONE 10, e0136365 (2015).

43. Tomato Genome, C. The tomato genome sequence provides insights into fleshy fruit evolution. Nature 485, 635-641 (2012).

44. Chen, $\mathrm{G}$. et al. Identification of a specific isoform of tomato lipoxygenase (TomloxC) involved in the generation of fatty acid-derived flavor compounds. Plant Physiol. 136, 2641-2651 (2004).

45. Zhang, Y. et al. Anthocyanin accumulation and transcriptional regulation of anthocyanin biosynthesis in purple bok choy (Brassica rapa var. chinensis). J. Agric. Food Chem. 62, 12366-12376 (2014).

46. Zhang, Y. et al. Genetically engineered anthocyanin pathway for high healthpromoting pigment production in eggplant. Mol. Breed. 36 (2016).

47. Wellburn, A. R. The spectral determination of Chlorophylls a and b, as well as total carotenoids, using various solvents with spectrophotometers of different resolution. J. Plant Physiol. 144, 307-313 (1994).

48. Exposito-Rodriguez, M., Borges, A. A., Borges-Perez, A. \& Perez, J. A. Selection of internal control genes for quantitative real-time RT-PCR studies during tomato development process. BMC Plant Biol. 8, 131 (2008).

49. Ha, J. H. et al. Nicotiana benthamiana Matrix Metalloprotease 1 (NMMP1) gene confers disease resistance to Phytophthora infestans in tobacco and potato plants. J. Plant Physiol. 218, 189-195 (2017).

50. Bayle, V., Nussaume, L. \& Bhat, R. A. Combination of novel green fluorescent protein mutant TSapphire and DsRed variant mOrange to set up a versatile in planta FRET-FLIM assay. Plant Physiol. 148, 51-60 (2008).

51. Albert, N. W. et al. A conserved network of transcriptional activators and repressors regulates anthocyanin pigmentation in eudicots. Plant Cell 26, 962-980 (2014).

52. An, J. P. et al. EIN3-LIKE1, MYB1, and ETHYLENE RESPONSE FACTOR3 act in a regulatory loop that synergistically modulates ethylene biosynthesis and anthocyanin accumulation. Plant Physiol. 178, 808-823 (2018).

53. Nguyen, C. V. et al. Tomato GOLDEN2-LIKE transcription factors reveal molecular gradients that function during fruit development and ripening. Plant Cell 26, 585-601 (2014).

54. Zhang, F., Gonzalez, A., Zhao, M., Payne, C. T. \& Lloyd, A. A network of redundant bHLH proteins functions in all TTG1-dependent pathways of Arabidopsis. Development 130, 4859-4869 (2003).

55. Ramsay, N. A. \& Glover, B. J. MYB-bHLH-WD40 protein complex and the evolution of cellular diversity. Trends Plant Sci. 10, 63-70 (2005).

56. Baudry, A., Caboche, M. \& Lepiniec, L. TT8 controls its own expression in a feedback regulation involving TTG1 and homologous MYB and bHLH factors, allowing a strong and cell-specific accumulation of flavonoids in Arabidopsis thaliana. Plant J. 46, 768-779 (2006).

57. Yamagishi, M. A novel R2R3-MYB transcription factor regulates light-mediated floral and vegetative anthocyanin pigmentation patterns in Lilium regale. Mol. Breed. 36 (2015).

58. Li, N. et al. Nonuniform gene expression pattern detected along the longitudinal axis in the matured rice leaf. Sci. Rep. 5, 8015 (2015).

59. Ostergaard, L. Don't 'leaf' now. The making of a fruit. Curr. Opin. Plant Biol. 12 36-41 (2009).

60. Meng, $X$. et al. Physiological changes in fruit ripening caused by overexpression of tomato SIAN2, an R2R3-MYB factor. Plant. Physiol. Biochem. 89, 24-30 (2015).

61. Schreiber, G. et al. ANTHOCYANIN1 from Solanum chilense is more efficient in accumulating anthocyanin metabolites than its Solanum lycopersicum counterpart in association with the ANTHOCYANIN FRUIT phenotype of tomato. Theor. Appl. Genet. 124, 295-307 (2012). 